\title{
Eshelby's solution for ellipsoidal inhomogeneous inclusions with applications to compaction bands
}

\author{
Chunfang Meng* ${ }^{*}$ David D. Pollard \\ Department of Geological and Environmental Sciences, Stanford University, Stanford, CA94305-2115, \\ U.S.A.
}

\begin{abstract}
Eshelby's solution for an ellipsoidal inhomogeneous inclusion in an infinite elastic body is applied to compaction and shear-enhanced compaction bands in the Aztec sandstone at Valley of Fire State Park, NV. The inclusion and matrix are linear elastic and isotropic, but have different elastic moduli, and a remote stress represents tectonic loading. A prescribed uniform strain within the inclusion accounts for inelastic compaction for a porosity change from $25 \%$ to $10 \%$. Differences in elastic moduli between the matrix and inclusion are based on laboratory data. We generalize earlier results, limited to 2D and axisymmetric geometries, by considering ellipsoids with different intermediate and greatest axial lengths, consistent with field observations.

Stiffness contrasts and non-circular tip-line shapes produce modest concentrations of the remote stress, but compaction strains of $1 \%$ to $10 \%$ produce significant triaxial compressive stress concentrations, which presumably are responsible for band propagation. The plastic strain is triaxial, but dominated by the normal strain across the inclusion. The stress diminution on the band flank is easily overcome by minor increases in the tectonic loading, enabling bands to be closely spaced. For the shear-enhanced band, if the plastic shear and normal strains are approximately equal, the ratio of shear to normal stress is about 1.3 at the tip.

Keywords: compaction band, shear-enhanced compaction band, Eshelby solution, ellipsoidal inhomogeneous inclusion, stress analysis
\end{abstract}

Email addresses: cf.meng@gmail.com (Chunfang Meng*), dpollard@stanford.edu (David D. Pollard)

Preprint submitted to Journal of Structural Geology

July 7, 2014

(C) 2014. This manuscript version is made available under the Elsevier user license http://www.elsevier.com/open-access/userlicense/1.0/ 
Compaction bands are one category of narrow, zones of deformation found in porous granular sedimentary rock: the broader classification of such structures is known as deformation bands (Aydin (1978)). Those deformation bands that formed primarily by shearing (usually with measurable shear offset of crossing markers in outcrop) are called shear bands; those formed primarily by volume reduction (with no measurable shear offset) are called compaction bands (Aydin et al. (2006)). A third category of the family of deformation bands is known as dilation bands Aydin et al. (2006). The occurrence of deformation bands is widespread across the U.S. Southwest (Davis (1999)), and they have been called "the most common strain localization feature found in deformed porous sandstones and sediments" (Fossen et al. (2007)). Their role in hydrocarbon reservoir permeability has been related to tectonic regime (Solum et al. (2012)), and microstructural characteristics (Ballas et al. (2012)). Here we focus on compaction bands (Mollema and Antonellini (1996)), but include some analysis relevant to bands that have been enhanced by shearing (Baud et al. (2004) and Eichhubl et al. (2010)).

The sub-parallel compaction bands (Eichhubl et al. (2004), Sternlof et al. (2005) and Aydin and Ahmadov (2009)) in Valley of Fire State Park, NV, are up to tens of meters in length (often terminated at dune boundaries), taper from up to $15 \mathrm{~mm}$ in thickness to zero at tips, and are spaced up to several meters apart. The Aztec Sandstone has a porosity of about $25 \%$ outside bands and about $10 \%$ inside bands. The bands have lower ${ }_{21}$ permeability than the surrounding matrix (Taylor and Pollard (2000) and Main et al. 22 (2001)), and as a result, compaction bands can significantly affect the performance of 23 sandstone reservoirs and aquifers (Sternlof et al. (2004), Sternlof et al. (2006) and Sun et al. (2011)).

In previous work (e.g. Katsman and Aharonov (2005), Katsman et al. (2006a) and spring network methods. Alternatively, the initiation of compaction bands has been modeled using bifurcation theory (e.g. Rudnicki and Rice (1975), Olsson (1999), Issen and Rudnicki (2000), Wong et al. (2001), Besuelle (2001), Borja and Aydin (2004), Rudnicki (2004) and Issen and Challa (2008)). In another conceptualization, compaction bands have been modeled using cracks and dislocations to exploit elastic fracture mechanics con- 
cepts (e.g. Rudnicki and Sternlof (2005), Rudnicki (2007), Schultz (2009), and Tembe

et al. (2008)). These theoretical studies have been motivated by field descriptions (e.g. Fossen et al. (2007), Mollema and Antonellini (1996), Eichhubl et al. (2004), Sternlof et al. (2005), Aydin and Ahmadov (2009) and Sternlof et al. (2006)) and compared to laboratory experiments on compaction phenomena in porous rock (e.g. Olsson (1999), Baud et al. (2000), Mair et al. (2000), Olsson and Holcomb (2000), Baud et al. (2004) and Vajdova and Wong (2003)).

To investigate the development and propagation of compaction bands, we focus on the inhomogeneous inclusion of Eshelby, which allows one to assess the stress perturbation inside and in the vicinity of a stiffer ellipsoidal body subjected to a prescribed compaction strain and remote stress. We use empirical relationships between porosity and elastic moduli along with measured porosities of compaction bands to specify the contrast in the elastic properties of the ellipsoidal inhomogeneity. Remote stress fields are based on the geologic and tectonic history of the Aztec Sandstone. The volumetric plastic strain for the inhomogeneous inclusion is scaled to the porosity loss.

The local stress perturbation is examined to understand the propensity for propagation along the elliptical tip-line of the inclusion and variations in the direction of pure compaction band propagation. The stress intensity for the plain strain 2D crack opened by a uniform remote tension is just $\pi / 2$ times that for the $3 \mathrm{D}$ penny-shaped crack (Tada et al. (2000). This proportional relation applies to opening displacement discontinuity as well. These results suggest, qualitatively, that varying the semi-axial ratio in the plane of the model compaction band will not have a large effect on the near tip stress field. We provide a quantitative assessment of this effect for the ellipsoidal inhomogeneity.

The true tip-line shape of compaction bands has not been recorded because of lack of exposure. However, very detailed mapping of dune architectures and trace lengths of bands over variable topography (Deng and Aydin (2012)) suggest that the tip-line shapes of compaction bands are likely to be eccentric rather than penny shaped. Eshelby's 3D solutions allow one to investigate the effects of varying axial ratios of an ellipsoidal inhomogeneity on the formation of a compaction band.

For shear-enhanced compaction bands, we scale the shear component of the plastic strain to the shear stress at the tip. The resulting plastic shear strain matches the field 
measurements (Eichhubl et al. (2010)).

\subsection{Eshelby's solutions}

Eshelby's solutions enable one to evaluate the quasi-static elastic fields (stress, strain and displacement) inside and outside an arbitrary ellipsoidal inclusion (same elastic moduli as surrounding) that has experienced a prescribed uniform strain, or an ellipsoidal inhomogeneous inclusion (different moduli than surroundings) subjected to a prescribed remote state of stress (Eshelby (1957), Eshelby (1959) and Eshelby (1961)). These solutions have been applied in studies of rock deformation associated with fractures in sedimentary strata (Reches (1998)), pebbles in a conglomerate (Eidelman and Reches (1992)), faulting (Rudnicki (1977)), localized volume reduction structures such as compaction bands (Sternlof et al. (2005)), and alterations of regional stress by a reservoir with different elastic constants than the surrounding rock (Rudnicki (1999)). Eshelby's solutions also have been used in modeling brittle fracture formation(Healy et al. (2006a), Healy et al. (2006b) and Meng et al. (2013)).

Because Eshelby's solutions are rather complex and lack analytical form for an arbitrary ellipsoid, researchers have degenerated the geometry to special shapes such that analytical solutions could be formulated. In previous research one of the ellipsoid's semiaxes was set much shorter than the other two that were set equal, i.e. a penny-shaped spheroid. Reches (1998) and Healy (2009) developed computer codes to evaluate the solution for spheroids. Recently, we developed a Matlab ${ }^{\mathrm{TM}}$ code for arbitrary 3D ellipsoidal geometries (Meng et al. (2011)). Because one axial length of the ellipsoidal model is very much less than the other two, the geometry approaches that of an embedded layer

(Cocco and Rice (2002)), which can be used to gain important insights about compaction bands (Sternlof et al. (2005)).

The inclusion problem described by Eshelby (1957) considers an ellipsoidal region in an infinite homogeneous, isotropic and elastic material known as the matrix (Fig. 1 A). When an inclusion undergoes a change in shape and size, its misfit with the surrounding matrix causes both regions to attain new strain/stress states. The definition of eigenstrain, $\epsilon^{*}$, summarized by Mura (1987), p. 1, can be regarded as a uniform strain state that the inclusion will enter if removed from the constraint of the matrix. Eshelby 
(1957) referred to this as stress-free strain. Eshelby solved for the elastic fields inside and outside the ellipsoidal inclusion for a given eigenstrain and zero remote stress.

We denote the sub-domain occupied by the inclusion $\Omega$ in the infinite domain $D$, and the uniform eigenstrain $\epsilon_{i j}^{*}$ is prescribed to be non-zero throughout $\Omega$ and zero in $D-\Omega$. The elastic moduli in the inclusion $\Omega$ and the matrix $D-\Omega$ are the same. The displacement, $u_{j}$, strain, $\epsilon_{i j}$ and stress, $\sigma_{i j}$ for both the inclusion and matrix are given by Mura (1987), p. 11.

The interior and exterior elastic strain fields for the inclusion problem (Eshelby (1957), Eshelby (1959) and Eshelby (1961)) are

$$
\begin{gathered}
\epsilon_{i j}=S_{i j k l} \epsilon_{k l}^{*}, \text { for } \mathbf{x} \in \Omega, \\
\epsilon_{i j}(\mathbf{x})=D_{i j k l}(\mathbf{x}) \epsilon_{k l}^{*}, \text { for } \mathbf{x} \in D-\Omega,
\end{gathered}
$$

where $S_{i j k l}$ is known as the Eshelby tensor, Mura (1987), p. $77 ; D_{i j k l}(\mathbf{x})$ in general involves some incomplete elliptical integrals. Meng et al. (2011) evaluated the elliptical integrals numerically with great precision using Igor (2005). Because of this the method is referred to as quasi-analytical.

\subsection{Equivalent inclusion method}

The other problem solved by Eshelby (Mura (1987)) considers the ellipsoidal region to have different elastic moduli, i.e. $C_{i j k l}^{*}$ in $\Omega$ and $C_{i j k l}$ in $D-\Omega$, and the body is subjected to remote uniform stress $\sigma_{i j}^{\infty}$ (Fig. 1B). This is referred to as the inhomogeneous inclusion problem. Eshelby (1957) concluded that the stress perturbation of a uniformly applied stress due to the presence of an ellipsoidal inhomogeneous inclusion can be determined by an inclusion problem when a fictitious eigenstrain $\epsilon_{i j}^{*}$ is chosen properly. This is known as the equivalent inclusion method.

To solve for $\epsilon_{i j}^{*}$, Meng et al. (2011) rewrites Mura (1987), 22.13:

$$
\left(\Delta C_{i j k l} S_{k l m n}-C_{i j m n}\right) \epsilon_{m n}^{*}=-\Delta C_{i j k l} \epsilon_{k l}^{\infty}-C_{i j k l}^{*} \epsilon_{k l}^{P},
$$

where $\Delta C_{i j k l}=C_{i j k l}-C_{i j k l}^{*}$; and from the relation $\sigma_{i j}^{\infty}=C_{i j k l} \epsilon_{k l}^{\infty}$, we have the remote strain $\epsilon_{i j}^{\infty} . \epsilon_{i j}^{p}$ is an arbitrary initial eigenstrain that may be prescribed for the ellipsoidal inhomogeneous inclusion. 
The interior and exterior stress and strain fields are as follows, Mura (1987), 22.822.13 :

$$
\begin{aligned}
\epsilon_{i j} & =\epsilon_{i j}^{\infty}+S_{i j m n} \epsilon_{m n}^{*}, \\
\sigma_{i j} & =\sigma_{i j}^{\infty}+C_{i j k l}\left(S_{k l m n} \epsilon_{m n}^{*}-\epsilon_{m n}^{*}\right), \text { for } x \in \Omega ; \\
\epsilon_{i j}(\mathbf{x}) & =\epsilon_{i j}^{\infty}+D_{i j m n}(\mathbf{x}) \epsilon_{m n}^{*}, \\
\sigma_{i j}(\mathbf{x}) & =\sigma_{i j}^{\infty}+C_{i j k l} D_{k l m n}(\mathbf{x}) \epsilon_{m n}^{*}, \text { for } \mathbf{x} \in D-\Omega .
\end{aligned}
$$

We model the compaction band with an ellipsoidal inhomogeneous inclusion that has axial-ratio $a_{x} \gg a_{z}$ and $a_{y} \gg a_{z}$, which gives suitable geometries for compaction bands (Mollema and Antonellini (1996), Sternlof et al. (2005), Eichhubl et al. (2010)). The elastic moduli of the inhomogeneous inclusion are related to the physical changes, e.g. porosity loss and grain size reduction, and in general they are different than the moduli of the matrix. The infinite elastic body is under a triaxial stress state with greatest principal compressive stress perpendicular to the $(x, y)$-plane of the highly eccentric ellipsoidal structure. For shear-enhanced compaction bands the greatest compressive stress is oblique to the band plane. The inelastic volume reduction is prescribed as the initial eigenstrain, $\epsilon_{i j}^{p}$. Analogous to Katsman et al. (2006b), we can set $\epsilon_{i j}^{p}$ to be uniaxial. In general however, e.g. when shearing across the band is clearly indicated, the initial eigenstrain is not uniaxial. Determining a realistic initial eigenstrain is addressed in Sections 4 and 4.2 .

\subsection{Compaction bands as tabular structures}

A compaction band as a tabular structure illustrated in Fig. $2 \mathbf{A}$ has been modeled in $2 \mathrm{D}$ by Katsman et al. (2006b). The prescribed uniaxial strain is uniform throughout a rectangular region of initial size $2 H \times 2 c$ such that $\epsilon_{z z}^{*}=(H-h) / H$. The remote stresses are zero, and the tabular structure has the same elastic moduli as the matrix. This is analogous to Eshelby's inclusion solution illustrated in Fig. 1 A, but the shape of the inclusion is rectangular not ellipsoidal. Katsman et al. (2006b) cites the laboratory experiments by Haimson (2003) and Baud et al. (2004) and concludes: these test results "demonstrate that compaction bands would have rectangular geometries." It has been found however, that both elliptical and bilinear approximations of the compaction band cross-section produce a better fit to field data (Sternlof et al. (2005) and Section 2.1). 
In comparing the $3 \mathrm{D}$ ellipsoidal inclusion and inhomogeneous inclusion models to the 2D tabular inclusion model, we set the parameters such that the 3D model emulates the 2D model: the uniaxial eigenstrain $\epsilon_{z z}^{*}=-(H-h) / H=-0.136$; the aspect ratio of the rectangular inclusion $H / c=8 \times 10^{-4}$; and the axial-ratios of the ellipsoid $a_{y} / a_{x}=$ $20, a_{z} / a_{x}=8 \times 10^{-4}$. Strictly speaking, $a_{y}$ should be infinite to match the $2 \mathrm{D}$ case, but we find as $a_{y} / a_{x} \geq 10$, the result does not vary significantly.

The rectangular and ellipsoidal inclusions have the same elastic moduli as the matrix, i.e. $C_{i j k l}=C_{i j k l}^{*}$, while the ellipsoidal inhomogeneous inclusion has different moduli. The elastic moduli are sampled from porosity-modulus relations for given porosities (see Section 2.2). In the inclusion cases, we let both inclusion and matrix have a porosity of $25 \%$. For the inhomogeneous inclusion case (see field data by Sternlof et al. (2005)), we set the porosities to $\phi_{i}=10 \%$ for the ellipsoid and $\phi_{m}=25 \%$ for the matrix. Similar to Sternlof et al. (2005) we set the remote stress $\sigma_{i i}^{\infty}=\left[-2 \times 10^{7},-2 \times 10^{7},-4 \times 10^{7}\right] \mathrm{Pa}$, where $i=x, y, z$.

Fig. 3 plots the normal stress $\sigma_{z z}$ along the $a_{x}$ axis for the 2D tabular model, elliptical inclusion model and elliptical inhomogeneous inclusion model. In both the $2 \mathrm{D}$ tabular and ellipsoidal inclusion cases the inside and outside stresses have opposite signs, tensile inside and compressive outside. Even though the prescribed plastic strain in the inclusion is stress free, the elastic matrix resists this uniaxial contraction and pulls back on the inclusion, inducing the tensile stress. The contraction of the inclusion causes a local contraction of the matrix distal from the tips, thereby inducing a compressive stress.

Unlike the 2D tabular inclusion, the elliptical inclusion has uniform inside stress given by Eq. (3). Also, the stress immediately distal from the tips is not singular. The $2 \mathrm{D}$ tabular region with a uniform uniaxial strain is equivalent to two edge dislocations (Pollard and Fletcher (2006) p. 305), where the near-tip normal stresses on either side of the dislocation line are concentrated as $\sigma_{i j} \propto r^{-1}$ and have opposite signs. In contrast, the stress at the tip of the ellipsoidal inclusion is finite due to the finite radius of curvature of the tip profile (see Section 5.3) .

For the ellipsoidal inhomogeneous inclusion, the stress is greater by a constant value relative to that of the ellipsoidal inclusion. The normal stress inside is less than the remote stress because the plastic deformation induces some stress relief, but it remains 
compressive. The near-tip stress is finite and more compressive than the remote stress.

Unlike the ellipsoidal inclusion, the ellipsoidal inhomogeneous inclusion is consistent with

the concept that the stress inside the compaction band should be compressive.

\section{Compaction bands as a stiffer ellipsoidal structures}

Based on the field data for single bands that show significant loss of porosity (Sternlof et al. (2005)), we suggest that the ellipsoidal inhomogeneous inclusion is more suitable for modeling compaction bands than tabular or ellipsoidal inclusions. In this section we review field data that document the cross-sectional shape of compaction bands and justify using the ellipsoidal shape. Also, we relate changes in elastic moduli to the porosity losses (Avseth et al. (2005)) and justify using Eshelby's inhomogeneous inclusion model.

\subsection{Cross-sectional shape of compaction bands: field data}

Sternlof et al. (2005) report data on the measured thickness distributions of sixteen compaction bands exposed tip to tip in outcrops of Astec Sandstone in the Valley of Fire State Park, NV. More than 1700 measurements on bands ranging in length from $1 \mathrm{~m}$ to $62 \mathrm{~m}$ show that a typical compaction band is thicker in the middle and tapers toward the tip. In Fig. 4, we apply elliptical and piece-wise linear fits to the thickness-length relations for eight measured bands. For some of the compaction bands (left column in Fig. 4), the elliptical fit performs better, with an average correlation coefficient 0.89 , while for some bands (right column in Fig. 4) the linear fit performs better, with an average correlation coefficient 0.86. Both elliptical and piece-wise linear fits perform significantly better statistically than the tabular shapes (Katsman et al. (2006b)), with uniform thickness approximated as the mean of measured values, yielding correlation coefficients of only about 0.15 .

At the edges, the tapered band profiles form rather pointed tips instead of round ones. One may related the bands' abrupt changes in width to the remote stress gradients, interaction with neighboring faults or bands and heterogeneous elastic moduli of the host rock. Still, we acknowledge that the misfit of the band profiles to the elliptical approximations at the tips would result in some stress/strain underestimation by the Eshelby model. 


\subsection{Elastic moduli of compaction bands and surroundings}

The compaction bands from the Astec Sandstone that were examined petrographically (Sternlof et al. (2005)) have a mean porosity of $11.9 \pm 3 \%$, whereas the sandstone outside the bands yields $24.5 \pm 2.9 \%$. This suggests that the rock inside the bands could have different elastic moduli than that outside (Gassmann (1951)). The elastic moduli (bulk modulus $K$, Young's modulus $E$ ) and Poisson's ratio $\nu$ as functions of porosity $\phi$ for dry (subscript $d$ ) and wet (subscript $w$ ) sandstones are (Avseth et al. (2005)):

$$
\begin{aligned}
& K_{d}=\frac{2 G\left(1+\nu_{d}\right)}{3\left(1-\nu_{d}\right)}, \text { where } G=G_{0}\left(1-\phi / \phi_{0}\right), \\
& K_{w}=K_{d}+\frac{1-\left(K_{d} / K_{0}\right)^{2}}{\phi / K_{l}+(1-\phi) / K_{0}-K_{d} / K_{0}^{2}}, \\
& \text { where } K_{0}=K_{d \mid \phi=\phi_{0}}, \\
& E_{d}=3 K_{d}\left(1-2 \nu_{d}\right), E_{w}=3 K_{d}\left(1-2 \nu_{d}\right), \\
& \nu_{d}=0.07, \nu_{w}=\frac{z-1}{1+2 z}, \text { where } z=\frac{3 K_{w}}{2 G},
\end{aligned}
$$

Here $\phi_{0}=0.4$ is the maximum porosity of the sandstone, $G_{0}=44 \mathrm{GPa}$ is the shear modulus of solid quartz, and $K_{l}=2.2 \mathrm{GPa}$ is the effective bulk modulus of water.

Fig. 5 plots the moduli and Poisson's ratio of wet sandstone as functions of porosity $\phi$. Given these variations in elastic moduli and the measured reductions in porosity, a compaction band is more appropriately modeled as an inhomogeneous inclusion problem, using the equivalent inclusion method (see Section 1.2), than as an inclusion problem. On the other hand, Sternlof et al. (2005) use the embedded layer model (Cocco and Rice (2002)) to show that the contrast in elastic moduli has a minor effect on the state of stress immediately adjacent to a compaction band in comparison to the effect due to the plastic strain. Rudnicki (2007) uses the Eshelby solution to reach a similar conclusion and show that the stress concentrates by a factor of 10 to 1000 times the far-field stress. Nevertheless, to be consistent with the known reduction in band porosity and associated increase in moduli, we retain the contrast in elastic properties for the Eshelby solution.

For demonstration purposes, we choose the curves of wet sandstone to sample the elastic moduli and Poisson's ratios for given porosities. As a result, the porosity change has an impact on both elastic moduli and Poisson's ratio. The process of propagation and development may produce variations of porosity reduction along compaction bands. 
If so, the laboratory data (Avseth et al. (2005)) and empirical relationships (Fig. 5 and Eq. (4)) suggest that the in-band elastic properties are likely to vary along the band, but such variations are ignored here.

In the following sections, we set the axial ratio, $a_{z}=10^{-3} \sqrt{a_{x} a_{y}}$ (given the thicknessto-length ratios measured by Sternlof et al. (2005)). The elastic moduli are sampled from Fig. 5 for porosities inside and outside the compaction band $\phi_{h}=10 \%$ and $\phi_{m}=25 \%$ respectively. The resulting elastic moduli are $E_{m}=37.31 \mathrm{GPa}, \nu_{m}=0.13$ for the matrix and $E_{h}=71.52 G P a, \nu_{h}=0.084$ for the inhomogeneous inclusion. Young's modulus for the inclusion is outside the range $(10 \sim 46 \mathrm{GPa})$ reported for sandstone (Bieniawski (1984)). We use these values to be consistent with Eq. (4) and (Fig. 5), but acknowledge that the Aztec Sandstone could have been considerably less stiff at the time the compaction bands formed. The curve extrapolation of Fig. 5 may result in some unusually low Poisson's ratios at low porosity, given that the Poisson's ratio for the inclusion, 0.084, is just outside the reported range $(0.1 \sim 0.4)$ for sandstone (Bieniawski (1984)).

\section{Near-tip stress state for the ellipsoidal inhomogeneity}

In order to evaluate the near-tip stress state we consider the flat ellipsoidal inhomogeneity with semi-axes $a_{x} \geq a_{y} \gg a_{z}$ and calculate the near-tip stress state at the end of the longest and intermediate semi-axes, $a_{x}$ and $a_{y}$, respectively. Following Sternlof et al. (2005) and Rudnicki (2007) continuity of the traction vector, $\mathbf{t}$, across the interface at the end of the longest semi-axis requires $t_{x}\left(a_{x}^{-}, 0,0\right)=-t_{x}\left(a_{x}^{+}, 0,0\right)$. The superscripts and + indicate points an infinitesimal distance on either side of the interface at $x=a_{x}$. If follows that the normal stress components acting perpendicular to the interface at those points are equal: $\sigma_{x x}\left(a_{x}^{-}, 0,0\right)=\sigma_{x x}\left(a_{x}^{+}, 0,0\right)$. To simplify the notation, we name the normal stress on the left side of this equation $\sigma_{x x}^{b}$, using the superscript $b$ to refer to the stress in the model compaction band at the designated point. We name the normal stress on the right side of this equation $\sigma_{x x}^{t}$, using the superscript $t$ to refer to the stress adjacent to the tip of the band in the matrix. By continuity of the traction and these definitions:

$$
\sigma_{x x}^{b}=\sigma_{x x}^{t} \text { at } x=a_{x}, y=z=0
$$



equal, so the tangential components of these displacements are equal. Using the same superscript notation as in Eq. (5) we have $u_{y}^{b}=u_{y}^{t}$ and $u_{z}^{b}=u_{z}^{t}$. It follows that spatial derivatives of these displacement components taken tangential to the interface must be continuous across the interface. These spatial derivatives are equivalent to the longitudinal strains parallel to the interface, so:

$$
\epsilon_{y y}^{b}=\epsilon_{y y}^{b} \text { and } \epsilon_{z z}^{b}=\epsilon_{z z}^{t} \text { at } x=a_{x}, y=z=0
$$

Hooke's Law for the isotropic linear elastic matrix provides relations among the strain and stress components at the tip in terms of the shear modulus, $\mu$, and Poissons ratio, $\nu$, for the matrix (Pollard and Fletcher (2006), eqs. 8.18, 8.26):

$$
\begin{gathered}
\epsilon_{y y}^{t}=1 /(2 \mu)\left[\sigma_{y y}^{t}-\nu\left(\sigma_{x x}^{t}+\sigma_{y y}^{t}+\sigma_{z z}^{t}\right) /(1+\nu)\right], \\
\epsilon_{z z}^{t}=1 /(2 \mu)\left[\sigma_{z z}^{t}-\nu\left(\sigma_{x x}^{t}+\sigma_{y y}^{t}+\sigma_{z z}^{t}\right) /(1+\nu)\right]
\end{gathered}
$$

Rearranging these equations to put known quantities on the left sides, and simplifying terms with Poissons ratio:

$$
\begin{aligned}
& 2 \mu(1+\nu) \epsilon_{y y}^{t}+\nu \sigma_{x x}^{t}=\sigma_{y y}^{t}-\nu \sigma_{z z}^{t} \\
& 2 \mu(1+\nu) \epsilon_{z z}^{t}+\nu \sigma_{x x}^{t}=-\nu \sigma_{y y}^{t}+\sigma_{z z}^{t}
\end{aligned}
$$

Employing the continuity conditions, Eq. (5) and (6), to eliminate the tip strains and stress from the left sides, and naming these known quantities $A$ and $B$, we have two equations in two unknowns, $\sigma_{y y}^{t}$ and $\sigma_{z z}^{t}$ :

$$
\begin{aligned}
& 2 \mu(1+\nu) \epsilon_{y y}^{b}+\nu \sigma_{x x}^{b}=A=\sigma_{y y}^{t}-\nu \sigma_{z z}^{t} \\
& 2 \mu(1+\nu) \epsilon_{z z}^{b}+\nu \sigma_{x x}^{b}=B=-\nu \sigma_{y y}^{t}+\sigma_{z z}^{t}
\end{aligned}
$$

Solving for the unknown stress components and using Eq (5), the three normal stress components are:

$$
\begin{aligned}
& \sigma_{x x}^{t}=\sigma_{x x}^{b}, \sigma_{y y}^{t}=\frac{A+\nu B}{1-\nu^{2}}, \\
& \sigma_{z z}^{t}=\frac{\nu A+B}{1-\nu^{2}}, \text { at } x=a_{x}^{+}, y=z=0
\end{aligned}
$$




$$
\text { stresses at the end of the intermediate semi-axis, where } x=z=0, y=a_{x}^{+} \text {. }
$$

To investigate the near-tip stress state we use model parameters from Rudnicki (2007) as modified from Sternlof et al. (2005) and in this paper. Youngs modulus and Poissons ratio for the matrix are $\mu_{b}=37.31 \times 10^{3} \mathrm{MPa}, \nu_{b}=0.13$, and these properties of the inhomogeneity are $\mu_{b}=71.52 \times 10^{3} \mathrm{MPa}, \nu_{b}=0.084$. The remote normal stresses are principal stresses: $\left[\begin{array}{ccc}\sigma_{x x}^{\infty} & \sigma_{y y}^{\infty} & \sigma_{z z}^{\infty}\end{array}\right]=\left[\begin{array}{lll}-20 & -20 & -40\end{array}\right] \mathrm{MPa}$. The plastic strain within the ellipsoid is that due to compaction in the $z$-direction, and we take this to vary over the range $0 \leq \epsilon_{z z}^{p}=-0.1$. For the penny-shaped band, $a_{x}=a_{y}=1, a_{z}=5 \times 10^{-4}$, with zero plastic strain the stress states in the band and at the tip are:

$$
\begin{aligned}
& {\left[\begin{array}{lll}
\sigma_{x x}^{b} & \sigma_{y y}^{b} & \sigma_{z z}^{b}
\end{array}\right]=\left[\begin{array}{lll}
-29.19 & -29.19-40.01
\end{array}\right] \mathrm{MPa}} \\
& {\left[\begin{array}{lll}
\sigma_{x x}^{t} & \sigma_{y y}^{t} & \sigma_{z z}^{t}
\end{array}\right]=\left[\begin{array}{lll}
-29.19 & -19.19-24.60
\end{array}\right] \mathrm{MPa}}
\end{aligned}
$$

The $x$ - and $y$-components of normal stress in the band are somewhat more compressive than the remotely applied stress, because the band is stiffer than the matrix. The $z$ component is nearly unchanged, because the band is so thin in that direction. At the tip the $x$-component matches that in the band, as anticipated from the first equation of Eq. (10), and is somewhat more compressive than the applied stress. Both the $y$ and $z$-components at the tip are somewhat less compressive than the applied stress, and less compressive than the band stress, because of the shielding effect of the stiffer band. Although the band is nearly twice as stiff as the matrix, the stress concentrations in the band due to this stiffness contrast are modest, because the band is so thin (Rudnicki (2007), Cocco and Rice (2002)). Although the aspect ratio of the band is very large, $a_{x} / a_{z}=2 \times 10^{3}$, the concentration and diminution of remotely applied stress at the tip are modest. This result is dramatically different than the significant near-tip stress concentration of an open $\operatorname{crack}$ (Meng et al. (2013)). That is, $\sigma_{z z}^{\text {tip }} / \sigma_{z z}^{\infty}$ would be in the order of $10^{3}$. The elastic stiffness of the material filling the model band greatly reduces closing displacements and therefore reduce the stress concentration.

In contrast to the modest effects of stiffness contrast and aspect ratio, the effect of the plastic strain can be very significant. In Fig. 6 the stress components normalized by their counterparts in the remote field are plotted versus the plastic strain for the same model parameters defined above. For reference, the normalized stress components at zero 
eigenstrain are: $\left[\sigma_{x x}^{t} / \sigma_{x x}^{\infty} \sigma_{y y}^{t} / \sigma_{y y}^{\infty} \sigma_{z z}^{t} / \sigma_{z z}^{\infty}\right]=[1.460 .960 .62]$. The $x$-component is somewhat enhanced, whereas the $y$-component is diminished slightly and the $z$-component is somewhat diminished.

Fig. 6 shows that as the magnitude of the plastic strain goes to $\epsilon_{z}^{\mathrm{p}}=-0.1$, the normalized $x$-component decreases slightly, the $y$-component increases to more than 25 times the remote stress, and the $z$-component increases to more than 95 times the remote stress: $\left[\sigma_{x x}^{t} / \sigma_{x x}^{\infty} \sigma_{y y}^{t} / \sigma_{y y}^{\infty} \sigma_{z z}^{t} / \sigma_{z z}^{\infty}\right]=\left[\begin{array}{ll}1.39 & 25.58 \\ 95.47\end{array}\right]$. A plastic strain of $10 \%$ within the model band overwhelms the near-tip stress changes due to the stiffness contrast and aspect ratio. The near-tip stress components are principal stresses and the state of stress is truly triaxial: $\left[\begin{array}{lll}\sigma_{1}^{t} & \sigma_{2}^{t} & \sigma_{3}^{t}\end{array}\right]=\left[\begin{array}{lll}-27.76 & -511.62-3818.80\end{array}\right] \mathrm{MPa}$.

This brings up a number of questions about the propagation of compaction bands. Since laboratory testing is generally done with axisymmetric loading, not true triaxial loading, how can we interpret laboratory data to provide insight about compaction bands? The plastic strain used here, and based upon conclusions of Sternlof et al. (2005), is uniaxial. Is uniaxial strain a likely outcome of true triaxial loading with principal stress differences, as in this example? This result also emphasizes the need to estimate the plastic strain, which we address in the next section.

\section{Plastic strain in compaction bands: a heuristic and iterative estimation}

The initial eigenstrain, $\epsilon_{i j}^{p}$, is defined as the plastic strain caused by the compaction, as revealed for example, by porosity losses from the field samples (Sternlof et al. (2005)). Resolving this plastic strain from the porosity change is not a straight forward matter. The three principal components $\epsilon_{i i}^{p}$ can be related to the porosity change by:

$$
\left(1+\epsilon_{x x}^{p}\right)\left(1+\epsilon_{y y}^{p}\right)\left(1+\epsilon_{z z}^{p}\right)=1-\left(\phi_{m}-\phi_{h}\right)
$$

To determine all six components of $\epsilon_{i j}^{p}$, we need five equations in addition to Eq. (12).

\subsection{Plastic strain in pure compaction bands}

Here, we focus on pure compaction bands, i.e. the ones formed without plastic shear deformation, Eichhubl et al. (2010). The strain components are $\epsilon_{i j}^{p}=0$, for $i \neq j$, which reduces the unknowns to three. Still, two additional equations are needed to resolve the triaxial strain. 
An approach in previous research, e.g. Katsman et al. (2006b) and Sternlof et al. (2005), is to assume a uniaxial plastic strain that is non-zero in the compaction direction, e.g. $\epsilon_{z z}^{p} \neq 0$ and the other components are zero. Eq. (12) is then reduced to:

$$
1+\epsilon_{z z}^{p}=1-\left(\phi_{m}-\phi_{h}\right) .
$$

For the parameters given in Section 2.2, the resulting uniaxial strain is $\epsilon_{z z}^{p}=-0.15$.

If we assume an isotropic triaxial strain such that $\epsilon_{x x}^{p}=\epsilon_{y y}^{p}=\epsilon_{z z}^{p}$, Eq. (12) becomes:

$$
\left(1+\epsilon_{z z}^{p}\right)^{3}=1-\left(\phi_{m}-\phi_{h}\right)
$$

The resulting triaxial strain is then $\epsilon_{x x}^{p}=\epsilon_{y y}^{p}=\epsilon_{z z}^{p}=-0.0527$.

The thin section photos of compaction bands (from Sternlof et al. (2005) and Eichhubl et al. (2010)) suggest that the plastic deformation is indeed triaxial, but more noticeable in the compaction $(z)$ direction, e.g. $\epsilon_{z z}^{p}<\epsilon_{x x}^{p} \approx \epsilon_{y y}^{p}<0$. Neither the uniaxial strain nor the isotropic triaxial strain can capture this plastic deformation thoroughly.

We propose an iterative algorithm that takes the tip-incipient stress and the plastic strain as input and output in a loop and seeks a convergent triaxial strain. The rationale (illustrated in Fig. 7) is explained as follows: As a compaction band forms and propagates, the tip-incipient matrix will join the band by undergoing the plastic strain. The tipincipient stress is responsible for this plastic strain.

Here, we formulate a heuristic relationships between the tip stress and the plastic strain. One simple relationship is truncated linear, by which we scale the magnitudes of three principal strains $\epsilon_{i i}^{p}$ as:

$$
\begin{aligned}
\epsilon_{x x}^{p} & =\epsilon_{z z}^{p} \frac{\bar{\sigma}_{x x}-\sigma^{c}}{\bar{\sigma}_{z z}-\sigma^{c}} H\left(\bar{\sigma}_{x x}-\sigma^{c}\right), \\
\epsilon_{y y}^{p} & =\epsilon_{z z}^{p} \frac{\bar{\sigma}_{y y}-\sigma^{c}}{\bar{\sigma}_{z z}-\sigma^{c}} H\left(\bar{\sigma}_{x x}-\sigma^{c}\right),
\end{aligned}
$$

where $\bar{\sigma}_{i i}$ are the average principal stresses at the band tip. $\sigma^{c}$ is the critical principal stress above which plastic compaction will happen, $H(\cdot)$ is a Heavyside function that makes the strain state zero when $\bar{\sigma}_{i i}<\sigma^{c}$.

We use average values of $N$ discrete tip-incipient points to approximate $\bar{\sigma}_{i j}$ by:

$$
\bar{\sigma}_{i j}=\frac{\oint \sigma_{i i} d s}{\oint d s} \approx \frac{\sum_{m}^{N} \sigma_{i j}^{(m)} \frac{2 \pi}{k_{m} N}}{\sum_{m}^{N} \frac{2 \pi}{k_{m} N}}=\frac{\sum_{m}^{N} \sigma_{i j}^{(m)} \frac{1}{k_{m}}}{\sum_{m}^{N} \frac{1}{k_{m}}}
$$


where $\sigma_{i j}^{(m)}$ and $k_{m}$ are tip-line stress and curvature of the $m$ th discrete point (Fig. 7).

With Eq. (12), Eq. (15) and Eq. (16), the principal plastic strains $\epsilon_{i i}^{p}$ can be resolved. When the new plastic strain is fed back to the model, a new tip stress is produced. By solving for $\epsilon_{i i}^{p}$ and $\sigma_{i j}$ iteratively we seek the convergent tip stress and plastic strain.

If we feed the new plastic strain directly to the iteration, the results will oscillate and never converge. Therefore, we combine the old plastic strain and the new one with a damping factor $\alpha \in[0,1]$. The flow diagram for such an algorithm is given in Fig. 8 .

We use the uniaxial strain and triaxial strain given by Eq. (13) and Eq. (14) as the initial guesses. For the model parameters given in Section 2.2 and damping factor $\alpha=0.3$, the convergent curves of the three principal strains are given in Fig. 9. The magnitude of critical stress, $\sigma^{c}$ is taken as $40 \mathrm{MPa}$, which is at the lower end of the reported range $(40 \sim 70 \mathrm{MPa})$ for sandstone (Bieniawski (1984)). The results suggest that no matter which initial condition we take, the strains always converge to the same three values:

$$
\left[\epsilon_{x x}^{p} \epsilon_{y y}^{p} \epsilon_{z z}^{p}\right]=[-0.012-0.015-0.127]
$$

The resulting plastic strain is triaxial with the normal component $\epsilon_{z z}^{p}$ greater than the other two principal components by a factor 10, suggesting this uniaxial component dominates the in-band plastic strain. The near-tip strain component $\epsilon_{z z}^{p}$ is not sensitive to the axial ratio $a_{y} / a_{x}$ of the ellipsoid.

\subsection{Plastic strain in shear-enhanced compaction bands}

We could scale the shear components of $\epsilon_{i j}^{p}$ linearly with the average tip shear stresses $\bar{\sigma}_{i j}$ that exceeds a critical shear stress, $\sigma_{\mathrm{sh}}^{c}$, similarly to Eq. (15). Unlike the principal plastic strains however, the shear strains do not affect the volume, and there is no analogous relation to Eq. (12) that would provide enough equations to resolve the shear strains. Instead, we simulate the shear deformation as an incremental process with a varying shear modulus. This allows one to relate the in-band plastic shear strain and the near-tip stress explicitly.

During the plastic shear deformation the effective shear modulus $G_{p}$ increases from the matrix shear modulus $G_{m}$ to the in-band shear modulus $G_{h}$ linearly with the plastic shear strain $\epsilon_{i j}^{p}$ for the remote stress rotated around the $y$-axis. Field measurements 
(Eichhubl et al. (2010)) suggest that the in-band plastic shear strain $\left(\epsilon_{x z}^{p}\right)$ has about the same magnitude as the plastic normal strain due to compaction $\left(\epsilon_{z z}^{p}\right)$. We let the effective shear modulus equal the final in-band shear modulus $\left(G_{p}=G_{h}\right)$ when $\epsilon_{x z}^{p}$ reaches $\epsilon_{z z}^{p}$.

We relate the in-band plastic shear strain to the near-tip stress as follow:

$$
\begin{aligned}
\epsilon_{i j}^{p(n)} & =\epsilon_{i j}^{p(n-1)}+\frac{\left(\bar{\sigma}_{i j}-\sigma_{\mathrm{sh}}^{c(n)}\right)}{G_{p}^{(n)}} H\left(\bar{\sigma}_{i j}-\sigma_{\mathrm{sh}}^{c(n)}\right), \\
\sigma_{\mathrm{sh}}^{c(n)} & =\sigma_{\mathrm{sh}}^{c(n-1)} \frac{G_{p}^{(n)}}{G_{p}^{(n-1)}}, \\
G_{p}^{(n)} & =G_{m}+\left(G_{h}-G_{m}\right) \frac{\epsilon_{i j}^{p(n)}}{\epsilon_{z z}^{p(n)}}, \text { for } i \neq j,
\end{aligned}
$$

where $n$ is the iteration number (see Section 4$) ; H(\cdot)$ is the Heavyside function. The initial values of the plastic shear strains $\epsilon_{i j}^{p(0)}$ (i.e. shear components of the eigenstrain) and the critical shear stress $\sigma_{\mathrm{sh}}^{c(0)}$ (for plastic shear deformation to happen) must be specified. We incorporate Eq. (18) into the iteration (Fig. 7 in Section 4) resolving all six components of the plastic strain $\epsilon_{i j}^{p}$.

The relative orientations between the pure and shear-enhanced compaction bands (Eichhubl et al. (2010)) suggest that the angle between the most compressive remote stress $\sigma_{3}^{\infty}$ and the normal vector of the shear-enhanced compaction band is about $45^{\circ}$ (Eichhubl et al. (2010) Fig. 18A).

We modify the example in Section 4 as follows: rotate the triaxial remote stress $\left(\sigma_{x x}^{\infty}=\sigma_{y y}^{\infty}=-20 \mathrm{MPa}, \sigma_{z z}^{\infty}=-40 \mathrm{MPa}\right)$ around the $y$-axis by $45^{\circ}$, which creates shear stress $\sigma_{x z}$ at the tip. The initial critical shear stress is $\sigma_{\mathrm{sh}}^{c(0)}=20\left(\sigma_{3}^{\infty}-\sigma_{1}^{\infty}\right) / 2$, i.e. twenty times the maximum remote shear stress, and we gradually increase the assumed initial shear strain $\epsilon_{x z}^{p(0)} \in[0.01,0.25]$. The resulting in-band strains $\epsilon_{i j}^{p}$ as functions of the iteration number $n$ are plotted in Fig. 19.

The three normal components of $\epsilon_{i j}^{p}$, similarly as in Fig. 9, quickly converge to constants, and the shear components, except $\epsilon_{x z}^{p}$, stay zero. When the initial value of the shear stain $\epsilon_{x z}^{p(n)}$ is small, it will not vary with $n$. This means that the shear stress at the tip is equal or less than the critical shear stress required for the initial plastic shear deformation. When the initial shear strain is large, it will increase with $n$ without becoming constant: the shear stress at the tip would trigger even larger in-band plastic shear than the initial one. 
The in-band plastic shear strain we seek for is the one that would cause the exact amount of tip-incipient stress which in turn would result in the same in-band plastic shear, i.e. the largest $\epsilon_{x z}^{p}$ that stays constant in Fig. 19. The observation that $\epsilon_{x z}^{p} \approx \epsilon_{z z}^{p}$ (Eichhubl et al. (2010)) is confirmed by the figure.

\section{Compaction bands under symmetric remote stress}

Arrays of sub-parallel to anastamosing compaction bands crop out in the upper half of the Aztec Sandstone in the Valley of Fire State Park (Fig. 10). Their outcrop traces vary in length from a few to more than $100 \mathrm{~m}$, and spacings vary from a few decimeters to more than $10 \mathrm{~m}$. In some cases the bands are clustered with spacings from zero (touching) to a few centimeters. Band segments may curve in proximity to adjacent segments forming eye shaped structures (Fig. 10 B). Despite these local irregularities, in many outcrops the bands form a systematic set with approximately parallel members. The approximate parallelism indicates that if the remote stress field at a scale larger than the band set is homogeneous, the bands were formed approximately parallel and perpendicular to the principal stresses. This motivates consideration of a model based on the Eshelby inhomogeneous inclusion under symmetric remote stresses.

In the following examples we investigate the ellipsoidal inhomogeneous inclusion with $a_{z} \ll a_{x}$ and $a_{z} \ll a_{y}$ subjected to a remote stress (Sternlof et al. (2005)) $\sigma_{i i}^{\infty}=$ $[-20,-20,-40] \mathrm{MPa}$, where $i=x, y, z$. The initial plastic strain $\left(\epsilon_{i j}^{p}\right)$ within the inclusion is determined by the iterative algorithm in Fig. 8 with all shear (off-diagonal) components zero. The elastic constants are determined as in Section 2.2 for the inclusion and matrix.

We evaluate the elastic fields in three different observation grids illustrated in Fig. 11. On the cross-section grid in the $(x-o-z)$ plane we illustrate how the stress is concentrated near the tip and diminished near the flank; in the $(x-o-y)$ plane we illustrate how the stress varies along the tip-line, and on the near-tip grid $\left(r-o^{\prime}-\lambda\right)$ we focus on the stress perturbation around the tip.

\subsection{Three-dimensionality of a pure compaction band}

In previous research, Healy (2009) and Reches (1998) evaluated the special case of the spheroidal inhomogeneities, where two of the three semi-axes are equal, $a_{x}=a_{y} \neq a_{z}$. 
Under symmetric remote stress states (principal stresses parallel to the semi-axes of the ellipsoid), the resulting stress is uniformly distributed along the circular tip line. The three-dimensionality of the ellipsoidal inhomogeneous inclusion allows one to investigate the stress variation along the tip when $a_{y} / a_{x} \neq 1$ (Meng et al. (2011)).

We plot the normalized major (compressive) principal stress $\sigma_{3} / \sigma_{3}^{\infty}$ for different axialratios $a_{y} / a_{x}=\left[\begin{array}{lll}0.5 & 1 & 2\end{array} 20\right]$ about the tip (at longitude $\theta=0$, i.e. an end of the semi-axis $a_{x}$ ) on the cross-section plane $y=0$ (first row in Fig. 12). As the ellipsoid stretches in the $a_{y}$ direction, the near-tip $(\theta=0)$ stress becomes more compressive, while the near-flank stress becomes less compressive. This indicates that the regions near a band tip are more likely to host new bands. Also, a band is somewhat more likely to propagate from the tip with a shorter axis (e.g. $a_{x}$ in the fourth column of Fig. 12). Furthermore, new parallel bands are more likely to form adjacent to a band with lesser axial ratio, because the region of stress diminution is smaller (e.g. first column Fig. 16).

We plot the normalized principal stress about the tip at $\theta=0$ on the circle $\left(o^{\prime}, r=\right.$ $10^{-2} \sqrt{a_{x} a_{y}}, \lambda$ ) (first row in Fig. 13). The bimodal distributed stress is symmetric in $\lambda$ for a symmetric remote stress. The stress concentration is less along the extension of the band $(\lambda=0)$ and increases to maxima to either side of the band plane $\left(\phi= \pm 61^{\circ}\right)$. The stress concentration is greater at the ends of the intermediate semi-axis (e.g. fourth column Fig. 12) than at the ends of the major semi-axis (e.g. first column Fig. 13). Note, when $a_{y} / a_{x}$ shifts from 0.5 to $2, a_{x}$ becomes the intermediate semi-axis, and $a_{y}$ becomes the major semi-axis.

\subsection{Influence region of a pure compaction band}

Because of the stress relieved by the plastic deformation (Fig. 12), the rock on the flanks of a pure compaction band is under less compressive stress than in the remote field, whereas in front of the tips it is under greater compression. The concentration immediately in front of the tip provides the stress required for propagation (see Section 4). Here we focus on the diminution to evaluate band spacing.

Where compaction band heights are much less than lengths, for example because they are confined by dune boundaries as illustrated in Fig. 14, the regions of diminution scale with the heights rather than the lengths. In Fig. 14 the bands are less than two meters in height, but tens of meters in length. In these instances the region of stress diminution 
may determine the spacing. In other examples bands are spaced more closely than even their shortest in-plane dimension. In these instances we must seek another explanation for spacing.

What increase in remote stress is necessary to promote the initiation and propagation of a closely spaced new band? For a new band to form parallel to the flank of an existing one, the compressive stress there must exceed the critical value $\sigma^{c}$. The close spacing of the sub-parallel compaction bands observed in the field (10) suggests that the stress diminution on a band flank must be overcome easily by an increasing tectonic stress.

We use a remote compressive stress acting normal to an existing band that increases in time to simulate an increase in tectonic compression, perhaps due to slip on the major thrust faults in the region. We suppose, for example, that the existing band does not propagate because its tip is constrained by dun boundaries. The principal stress $\sigma_{z z}$ along the $z$-axis under increasing $\sigma_{z z}^{\infty}$ is plotted in Fig. 15. When the normal remote stress becomes slightly more compressive, e.g. by $10 \%$, the compaction condition $\sigma_{z z}=\sigma^{c}$ comes much closer to the band flank. This indicates that while the strain accommodation capability of a single compaction band is quickly consumed in a tectonic process, a closely spaced parallel band (or band set) may form to further accommodate the remote stress build up.

\subsection{Propagation of a pure compaction band}

It seems reasonable to postulate that compaction bands propagate along a path that remains perpendicular to the local greatest compressive stress, $\sigma_{3}$. We investigate propagation under symmetric loading based on this near-tip principal stress (first rows of Fig. 12 and Fig. 13). The bimodal near-tip stress distribution is similar to that of a mode I crack (Williams (1957) and Lawn and Wilshaw (1975), P.55), but of opposite sign (compressive instead of tensile).

The bimodal distribution raises a question: why do compaction bands choose not to branch following the two maximum compression planes, but rather propagate in between these planes along a more-or-less straight path $(\lambda=0)$ ? This can be explained by asserting that a compaction band can only propagate from its tip. This assertion has been employed (e.g. Erdogan and Sih (1963)) in studies of mixed-mode I II crack propagation. 
Lawn and Wilshaw (1975), Fig. 3.5 show that a mode I crack tip, analogous to pure compaction bands, has the circumferential stress $\sigma_{\lambda \lambda}$ peaking at $\lambda=0$.

For each instance of the principal (most compressive) stress $\sigma_{3}$ evaluated on a circle about the tip (Fig. 11B) using the polar coordinates $\left(o^{\prime}, r, \lambda\right)$, there is a plane $L$ passing through the evaluation point and perpendicular to $\sigma_{3}$. Among these planes there is a special one, in between the two planes associated with the two peak compressive stresses, that contains the origin $o^{\prime}$. In other words $\left|o^{\prime} o^{\prime \prime}\right|=0$, where $o^{\prime \prime}$ is the perpendicular projection of $o^{\prime}$ on the plane $L$. By the forgoing assertion, this special plane contains the next increment of the propagating band originated from $o^{\prime}$. For symmetric loadings the special plane aligns with the plane of the band, i.e. the $x-o-y$ plane. Deviation from this path could be caused by local heterogeneity of the rock properties (e.g. cross bedding, dune boundaries and other bands) or changes in the local stress orientations due to mechanical interaction with nearby bands.

While the propagation criterion suggested here is consistent with sub-parallel arrays of compaction bands (Rudnicki (2007), Sternlof et al. (2006)), the fact that the circumferential stress is not the greatest compression in the near-tip field is problematic. The micro-mechanical mechanisms operating at the band tip that would favor propagation perpendicular to a lesser compression have not been elucidated.

\section{Compaction bands under asymmetric remote stresses}

Under symmetric remote stress, a pure compaction band, once formed, will tend to propagate in plane, as described in Section 5.3. Field observations suggest however, that some bands offset the sedimentary layering or older bands, indicating they accommodated some shearing (Eichhubl et al. (2010)). This brings up the possibility that in the process of compaction band formation and propagation, the plane on which the major principal (compressive) stress acts would not align well with the band plane. We model this miss alignment by rotating the remote stress $\sigma_{i j}^{\infty}$ around the $y$-axis by an angle $\eta_{y}$. Local stress perturbations on scale of 1 to 10 meters would provide more realistic conditions for this miss alignment, however this is beyond capability of Eshelby's solution.

We assume that a compaction band already is formed within the major principal stress plane, and its plastic strain $\epsilon_{i j}^{p}$ does not change with the remote stress rotations. 
Thus, we let the band have a fixed plastic strain generated under the symmetric remote stresses (produced by the iteration scheme shown in Fig. 8). For this plastic strain and different rotation angles $\eta_{y}$, we evaluate the near-tip stress fields and try to relate them to observed band geometries. This addresses the possible change in propagation direction of a pure compaction band that experiences a change in the remote stress orientation.

In addition to the model parameters given in Section 2.2, we investigate the case of an axial ratio $a_{y} / a_{x}=0.5$ in more detail. The remote stress rotation around the $y$-axis would create the local stress at $\left[\begin{array}{lll}a_{x} & 0 & 0\end{array}\right]$ analogous to a crack tip under mixed mode I II loading (Lawn and Wilshaw (1975), Chapter 3).

\subsection{Three-dimensionality of a shear-enhanced compaction band}

Similarly to the symmetric case, we plot the near-tip normalized principal stresses under different asymmetric loadings, i.e. for the rotation angle $\eta_{y}=[15,30,45]^{\circ}$, on the Cartesian and circular coordinates (Fig. 11). The stress distributions on the cross section plane $y=0$ (Fig. 12 second through fourth rows) tilt following the remote stress rotation, and the magnitudes slightly decrease. This indicates that under asymmetric stresses a compaction band would tend to propagate off-plane, or stop due to the lesser stress.

The tip stress variations are also shown on the polar coordinates (Fig. 13 second through fourth rows). The most compressive stress distribution shifts toward in positive $\lambda$ with the remote stress rotation.

For $a_{y} / a_{x}=0.5$, we zoom in to the tip at $\theta=0$ and plot the normalized principal stress $\sigma_{3} / \sigma_{3}^{\infty}$ (first column in Fig. 16). To have a quantitative indication of the off-plane growth tendency we plot the normalized circumferential stress $\sigma_{\lambda \lambda} / \sigma_{3}^{\infty}$ with respected to the tip tangent vector $n_{t}=\left[\begin{array}{lll}0 & 0 & 1\end{array}\right]$ (second column in Fig. 16). The stress contour tilts following the rotation. This is consistent with an off-plane growth tendency which is better indicated by the maximum traces of the contours of circumferential stress $\sigma_{\lambda \lambda}$ such that the off-plane angle is roughly equal to $\eta_{y}$.

\subsection{Propagation of shear-enhanced compaction band}

If we analyze the near-tip stress analogous to a mode I II crack (see Erdogan and Sih (1963)), the band, with certain conditions met, will propagate perpendicular to the 
maximum circumferential stress. For example the tip (second and third rows in Fig. 16)

would kink along the maximum trace of the $\sigma_{\lambda \lambda}$ contours.

The fact that the tip-incipient material undergoes a plastic strain and joins the compaction band, and that the stress along the tip-line is finite, motivate a propagation criterion different than the classic fracture mechanics criterion for a crack.

Eichhubl et al. (2010) suggest that shear stress may enhance the compaction band formation. In Fig. 17, we plot the near-tip maximum shear stress $\left(\sigma_{3}-\sigma_{1}\right) / 2$ and circumferential shear stress $\left(\sigma_{r \lambda}\right)$ normalized by the remote maximum shear stress on the near-tip grid (at longitude $\theta=0$ ). The maximum shear stress has distributions similar to the major principal stress in Fig. 16 (comparing the first columns).

When a compaction band forms, the band plane may not be aligned precisely with the plane normal to the greatest compression because the Aztec Sandstone is anisotropic due to cross bed layering, and it is heterogeneous due to sand dune boundaries. As a result, the stress $\sigma_{\lambda \lambda}\left(\sigma_{r \lambda}\right)$ will not be symmetric (anti-symmetric), e.g. about $a_{x}$ in Fig. 16 (Fig. 17). For example, one of the two anti-symmetric wings of $\sigma_{r \lambda}$ becomes weaker. This, combined with a kinked $\sigma_{\lambda \lambda}$, could enhance the compaction about the other stronger wing.

If the shear stress indeed enhances the compaction deformation, the direction in which the band tip would advance off-plane is likely to be in between the maximum trace of $\sigma_{\lambda \lambda}$ and the stronger wing of $\sigma_{r \lambda}$. This leads to a kink angle that overshoots the stress rotation angle $\eta_{y}$. When the new tip grows, the remote stress may correct this overshoot by turning the tip back toward its former orientation.

The stress fields calculated using Eshelby's inhomogeneous inclusion combined with some propagation criteria can be used to predict the incipient direction of out-of-plane propagation. Unfortunately because of its geometrical limitation, if the compaction band develops into some non-ellipsoidal geometry, Eshelby's solution will cease to apply.

\section{Stress condition for shear-enhanced compaction bands}

Plastic shear deformation is inferred by Eichhubl et al. (2010) along some planar compaction bands, based on offset bedding and force chains. Geometric relationships among shear-enhanced compaction bands suggest that two orientations of these bands, 
presumably symmetric and oblique to the principle stress plane, developed at the same time (Fig. 18). In particular, wavy bands, exhibiting two alternating orientations, join shear-enhanced bands of one or the other orientation (Fig. 18A, B). Also, individual bands from one shear-enhanced set can be traced continuously through a kinked path to become parallel to the second set, and vice versa (Fig. 18). The in-band plastic shear strain and normal strain are both about $10 \%$ as inferred by Eichhubl et al. (2010). In Section 4.2, an iterative method is used to find the in-band plastic shear, and the result is consistent with Eichhubl et al. (2010).

Here, we focus on the tip-incipient stress that would cause the combination of shear and compaction. We impose the relation $\epsilon_{x z}^{p} / \epsilon_{z z}^{p}=c$ for $c \in[0.11 .5]$ and set the remote stress the same as in Section 4.2, i.e. $\sigma_{3}^{\infty}$ at $45^{\circ}$ to the band. A penny-shaped band shape is assumed in addition to other parameters as in Section 2.2.

The resulting shear stress $\bar{\sigma}_{x z}$ and stress ratio $\bar{\sigma}_{x z} / \bar{\sigma}_{z z}$ are linearly related to the strain ratio $\epsilon_{x z}^{p} / \epsilon_{z z}^{p}$. If (as mentioned by Eichhubl et al. (2010)) $\epsilon_{x z}^{p} / \epsilon_{z z}^{p} \approx 1$, the corresponding tip stress ratio $\bar{\sigma}_{x z} / \bar{\sigma}_{z z}$ is approximately 1.3 .

\section{Discussion and conclusion}

Eshelby's solution for the ellipsoidal inhomogeneous inclusion offers many insights about the mechanics of localized compaction in porous rock. Here, we have focused on applications to compaction and shear-enhanced compaction bands. Attributes of this solution include 3D geometry of the inclusion, stiffness contrast between inclusion and matrix, remote triaxial stress, compaction eigenstrain within the inclusion, and nonsingular stress along the tipline. Further enhancements to this idealized model that could provide additional insights include non-ellipsoidal geometry of the inclusion, and spatially varying elastic moduli and eigenstrain within the inclusion. Varying the aspect ratio $\left(a_{y} / a_{x}\right)$ in the plane of the inclusion enables us to investigate the formation of compaction bands in 3D. The intermediate axial length of compaction bands (usually the vertical axis due to constraints of the dune boundaries) controls the spacing of bands. The increase in remote tectonic stress necessary to initiate a nearby band is modest.

The compaction bands observed and mapped at Valley of Fire, NV are depicted as highly eccentric ellipsoids, which show more resemblance to the field observations 
than the tabular inclusion model. Also, the inhomogeneous inclusion model keeps inband stresses compressive, unlike the homogeneous inclusion, where the in-band normal stress is tensile. The moduli contrast and the large aspect ratio produce modest stress concentration at the tip, in contrast to the open crack where this stress scales with the aspect ratio. The maximum compressive stress has a bimodal distribution around the tip, which begs the question: why do compaction bands propagate in plane? A new propagation criterion is consistent with in-plane propagation, but leaves an unresolved micro-mechanical mechanism that operates at the band tip. Further studies are needed to explain why some compaction bands have more tapered profiles than others, and to investigate the mechanical interactions of neighboring bands and the response of bands to heterogeneous and anisotropic mechanical properties of the host rock.

We formulate truncated linear relationships between the plastic strains and the tipincipient stresses, Eq. (15) and Eq. (18). These heuristic relationships are subject to improvements, as one further explores the plastic stress-strain relationships, but they admit an estimation of the plastic strain state within the band, which is required as input for the Eshelby inhomogeneous inclusion model. Although the dominant stress at the tip is that acting across the plane of the band, the stress state is triaxial. This raises doubts about the presumption that the eigenstrain within the band is uniaxial. In this regard, further characterization of the deformation within bands based on microscopic analysis and force chain construction are in order. This model is consistent with a shear-compression stress ratio $(c=1.3)$ under which shear-enhanced compaction bands are favored. The continuous joining in outcrop of two nearly orthogonal sets of shearenhanced bands with one symmetric set of wavy bands provides evidence that they all developed under the same loading conditions. This observation constrains possible propagation mechanisms, but the Eshelby solution is not adequate to investigate nonplanar and interacting bands. 


\section{Appendices}

\section{A. Validation of Matlab ${ }^{\mathrm{TM}}$ Code}

The Matlab ${ }^{\mathrm{TM}}$ code developed by Meng et al. (2011) evaluates the solutions by Eshelby for the quasi-static linear elastic fields (stress, strain, and displacement) inside an ellipsoidal body with arbitrary semi-axial lengths $\left(a_{x} \geq a_{y} \geq a_{z}\right)$ and in the surrounding infinite elastic body (Eshelby (1957), Eshelby (1959) and Eshelby (1961)). The ellipsoid and the infinite body each are homogeneous and isotropic with respect to elastic moduli, but may have different moduli, and the interface between them is perfectly bonded. Here we compare results from the code to analytical equations given by Mura (1987) for two special cases, the sphere and the penny-shaped inclusion. Also, we compare the code to analytical results given by Rudnicki (2007) for the axisymmetric inhomogeneity, which is used as a model for compaction bands. Other validations of the code are provided by Meng et al. (2011).

\section{A.1. Ellipsoidal inclusion approaching a spherical shape}

For inclusion problems the elastic moduli of the ellipsoid and the matrix are identical: the entire elastic domain is homogeneous with respect to these material properties. Young's modulus is taken as $1 \mathrm{GPa}$ and Poissons ratio is 0.1 for this example. Deformation is induced in the matrix by a uniform stress-free strain (the eigenstrain) within the inclusion. The components of the eigenstrain are prescribed as:

$$
\epsilon_{i j}^{*}=0.01, \quad i, j=x, y, z
$$

However, the elastic resistance to deformation of the matrix constrains the inclusion from achieving this deformation, so the final strain there is somewhat different than Eq. (19), and this constraint induces a uniform stress within the inclusion, which is calculated here. The stress, strain, and displacement fields in the matrix are inhomogeneous, and all components go to zero at an infinite distance. From Mura (1987) (Eq. 11.21.1) the normal stress component, $\sigma_{x x}^{s}$, inside the spherical inclusion with semi-axes $a_{x}=a_{y}=a_{z}$ is:

$$
\sigma_{x x}^{s}=\frac{2 \mu}{15(1-\nu)}\left[-8 \epsilon_{z z}^{*}-(5 \nu+1)\left(\epsilon_{y y}^{*}+\epsilon_{z z}^{*}\right)\right]
$$


Here $\mu$ is the elastic shear modulus and $\nu$ is Poissons ratio. The two other normal stress components are found by cycling the subscripts. The shear stress component, $\sigma_{x y}^{s}$, inside the spherical inclusion is:

$$
\sigma_{x y}^{s}=-\frac{2 \mu(7-5 \nu)}{15(1-\nu)} \epsilon_{x y}^{*}
$$

The two other shear stress components are found by cycling the subscripts.

In Fig. 20 we plot the stress components inside the ellipsoidal inclusion, $\sigma_{i j}^{E}$, each normalized by the respective stress component for the spherical inclusion, $\sigma_{i j}^{S}$. The semiaxis $a_{x}$ is fixed and the other two are equal and less than $a_{x}$. The normalized stress components are plotted versus the difference between the unequal semi-axes normalized by $a_{x}$, that is $\left(a_{x}-a_{y}\right) / a_{x}$ and $\left(a_{x}-a_{z}\right) / a_{x}$. As these differences decrease the shape of the ellipsoid approaches that of the sphere and the normalized stresses approach unity. When the normalized axial differences are less than $2.5 \times 10^{-2}$, the ellipsoidal stress components are within $1 \%$ of those for the sphere. For values of the normalized axial difference equal to $1 \times 10^{-4}$ the ellipsoidal stress components are within $0.004 \%$ of those for the sphere. For lesser axial differences the code becomes numerically unstable, so the calculated stress components are replaced with the analytical values for the sphere from Eq. (20), Eq. (21), and their cyclic permutations.

\section{A.2. Ellipsoidal inclusion approaching a penny-shape}

The penny-shaped ellipsoidal inclusion is configured such that the semi-axes are related as $a_{x}=a_{y} \gg a_{z}$. The elastic moduli of the ellipsoid and the matrix are identical and deformation is induced by a prescribed uniform eigenstrain $\epsilon_{i j}^{*}$ within the inclusion (Eq. (19)). The stress, strain, and displacement components go to zero at an infinite distance. By Mura (1987), Eq. 11.23.3, the stress components in the plane of the pennyshaped inclusion in the limit $a_{z} \rightarrow 0$ are:

$$
\begin{aligned}
& \sigma_{x x}^{P}=-2 \mu\left[\frac{\nu\left(\epsilon_{x x}^{*}+\epsilon_{y y}^{*}\right)}{1-\nu}+\epsilon_{x x}^{*}\right], \\
& \sigma_{x y}^{P}=-2 \mu \epsilon_{x y}^{*}, \\
& \sigma_{y y}^{P}=-2 \mu\left[\frac{\nu\left(\epsilon_{x x}^{*}+\epsilon_{y y}^{*}\right)}{1-\nu}+\epsilon_{y y}^{*}\right] .
\end{aligned}
$$

The stress components out of the plane of this penny-shaped inclusion are:

$$
\sigma_{x z}^{P}=\sigma_{y z}^{P}=\sigma_{z z}^{P}=0
$$


This is called a flat penny-shaped inclusion.

In Fig. 21 we plot the stress components $\sigma_{x x}^{E}, \sigma_{x y}^{E}$ and $\sigma_{y y}^{E}$ acting in the $(x, y)$ plane inside the ellipsoidal inclusion, each normalized by the respective stress component for the penny-shaped inclusion, $\sigma_{i j}^{P}$, from Eq. (22). The semi-axis $a_{x}$ is fixed, and the normalized difference in the semi-axes $\left(a_{x}-a_{y}\right) / a_{z}$ and the normalized minor axis $a_{z} / a_{x}$ are made equal and both diminished toward zero. As these quantities decrease the shape of the ellipsoidal inclusion approaches that of the flat penny-shaped inclusion and the normalized stresses approach unity. When the normalized minor axis is less than $6 \times 10^{-3}$, the ellipsoidal stress components are within $1 \%$ of those for the flat pennyshaped inclusion from Eq. (22).

In Fig. 22 we plot the normalized stress components $\sigma_{x z}^{E}, \sigma_{y z}^{E}$ and $\sigma_{z z}^{E}$ acting in the $z$-direction inside the ellipsoidal inclusion. Because the respective stress components for the penny-shaped inclusion are zero in the limit as $a_{z} \rightarrow 0$, we normalize the components for the ellipsoidal inclusion by $2 \mu \epsilon_{i z}^{E}$. Again, the semi-axis $a_{x}$ is fixed, and the normalized difference in the semi-axes $\left(a_{x}-a_{y}\right) / a_{x}$ and the normalized minor axis $a_{z} / a_{x}$ are made equal and diminished toward zero. As these quantities decrease the shape of the ellipsoidal inclusion approaches that of the flat penny-shaped inclusion and the normalized stresses approach zero.

\section{A.3. Axisymmetric inhomogeneous inclusion}

Rudnicki (2007) has investigated the stress state within the axisymmetric inhomogeneity, $a_{x}=a_{y} \gg a_{z}$, as a model for compaction bands. This solution combines the inclusion, for which deformation is induced by a stress-free eigenstrain, with the deformation induced by remotely applied stresses. The eigenstrain simulates the compaction within the band as induced by a tectonic compression. In addition, the elastic moduli inside the ellipsoidal may differ from those in the matrix. Here we graphically compare these analytical solutions to those found using the Matlab ${ }^{\mathrm{TM}}$ code.

To evaluate the effect of varying the eigenstrain and the aspect ratio of the pennyshaped ellipsoid, Rudnicki (2007) follows Sternlof et al. (2005) and takes Youngs modulus as $20 \mathrm{GPa}$ and Poissons ratio as 0.2 for both the ellipsoid and the matrix. In other words the elastic properties are homogeneous throughout. The remote stress state is $\left[\begin{array}{llllll}\sigma_{x x}^{\infty} & \sigma_{x y}^{\infty} & \sigma_{x z}^{\infty} & \sigma_{y y}^{\infty} & \sigma_{y z}^{\infty} & \sigma_{z z}^{\infty}\end{array}\right]=\left[\begin{array}{llllll}-20 & 0 & 0 & -20 & 0 & -40\end{array}\right]$ MPa. The ellipsoid 
is symmetrically loaded because these normal stresses are principal stresses, and the greatest compressive stress, $\sigma_{z z}^{\infty}$, is perpendicular to the band. The eigenstrain components all are zero except the normal component in the $z$-direction, which is varied as $\sigma_{z z}^{\infty} /\left(2 \mu \epsilon_{z z}^{\infty}\right)=\left[\begin{array}{lll}0.20 & 0.05 & 0.01\end{array}\right]$. Given constant remote stress and shear modulus, the eigenstrain is $\left[\begin{array}{lll}\epsilon_{x x}^{p} & \epsilon_{y y}^{p} & \epsilon_{z z}^{p}\end{array}\right]=\left[\begin{array}{lll}-0.012-0.048-0.240\end{array}\right]$. These values span the value suggested by Sternlof et al. (2005) from measurements of porosity loss for compaction bands in the Aztec sandstone at the Valley of Fire, NV.

In Fig. 23 the normalized stress acting across the model compaction band, $\sigma_{z z}^{i} / \sigma_{z z}^{\infty}$, is plotted versus the $\log$ of the aspect ratio, $\log \left(a_{z} / a_{x}\right)$. This plot is indistinguishable from Rudnicki (2007) (Fig. 2a). For aspect ratios less than about $1 \times 10^{-3}$ the stress in the ellipsoid is nearly equal to the remotely applied stress, which is the state of stress in the limit as $a_{z} / a_{x} \rightarrow 0$. Note that at greater aspect ratios the normal stress changes sign for the greater values of prescribed eigenstrain. That is, the specified combination of a remotely applied compression and contractional eigenstrain result in a tensile stress within the band. This seemly non-intuitive result is caused by the elastic reaction of the matrix to the stress free contractional eigenstrain and the boundary condition of perfect bonding. In reaction to the contraction, the matrix pulls back on the ellipsoid and induces the tensile stress. However, measured aspect ratios for compaction ands (Sternlof et al. (2005)) are about $10^{-4}$, so one should expect compressive stress states within natural compaction bands.

To evaluate the effect of varying the elastic modulus of the ellipsoid, Rudnicki (2007) takes $\mu^{i} / \mu=[0.1,1,10]$ and Poissons ratio as 0.2 for both the ellipsoid and the matrix. This is the inhomogeneous inclusion problem and the range of shear modulus ratios spans those likely to occur in nature. For this example $\sigma_{z z}^{\infty} /\left(2 \mu \epsilon_{z z}^{\infty}\right)=0.05$ and all other parameters are the same as in the previous example. In Fig. 24 the normalized stress acting across the model compaction band, $\sigma_{z z}^{i} / \sigma_{z z}^{\infty}$, is plotted versus the log of the aspect ratio, $\log \left(a_{z} / a_{x}\right)$. This plot is indistinguishable from Rudnicki (2007) (Fig. 2b). For aspect ratios less than about $10^{-3}$ the stress in the ellipsoid is nearly equal to the remotely applied stress, which is the state of stress in the limit as $a_{z} / a_{x} \rightarrow 0$. For larger aspect ratios the normalized stress decreases and becomes negative for $a_{z} / a_{x}>5 \times 10^{-2}$. Again, this tensile stress is unlikely to occur in nature where the aspect ratios are considerably 
smaller. Changing the modulus ratio over two orders of magnitude has very little effect

on the stress within the inhomogeneity.

Acknowledgments: We thank Atilla Aydin for introducing us to compaction bands in the field; Peter Eichhubl for the concept of shear-enhanced compaction bands; Kurt

Sternlof and John Rudnicki for field and theoretical guidance with respect to compaction bands. This research was supported by grant DE-FG02-04ER15588 from the Department of Energy, Basic Energy Sciences Program and the Stanford University Rock Fracture Project.

\section{References}

A. Aydin, Small faults formed as deformation bands in sandstone, Pure and Applied Geophysics 116 (1978) 913-930. 10.1007/BF00876546.

A. Aydin, R. I. Borja, P. Eichhubl, Geological and mathematical framework for failure modes in granular rock, Journal of Structural Geology 28 (2006) $83-98$.

G. H. Davis, Structural geology of the Colorado Plateau region of southern Utah, with special emphasis on deformation bands, Boulder, Colo. : Geological Society of America, 1999.

H. Fossen, R. A. Schultz, Z. K. Shipton, K. Mair, Deformation bands in sandstone: a review, Journal of the Geological Society 164 (2007) 755-769.

J. G. Solum, J. P. Brandenburg, S. J. Naruk, Characterization of deformation bands associated with normal and reverse stress states in the Navajo Sandstone, Utah: Reply, AAPG Bulletin 96 (May 2012) 877-890.

G. Ballas, R. Soliva, J.-P. Sizun, A. Benedicto, T. Cavailhes, S. Raynaud, The importance of the degree of cataclasis in shear bands for fluid flow in porous sandstone, American Association of Petroleum Geologists (2012).

P. Mollema, M. Antonellini, Compaction bands: a structural analog for anti-mode I cracks in aeolian sandstone, Tectonophysics 267 (1996) $209-228$.

P. Baud, E. Klein, T.-F. Wong, Compaction localization in porous sandstones: spatial evolution of damage and acoustic emission activity, Journal of Structural Geology 26 (2004) 603 - 624.

P. Eichhubl, J. N. Hooker, S. E. Laubach, Pure and shear-enhanced compaction bands in Aztec Sandstone, Journal of Structural Geology 32 (2010) 1873 - 1886.

P. Eichhubl, W. L. Taylor, D. D. Pollard, A. Aydin, Paleo-fluid flow and deformation in the Aztec Sandstone at the valley of fire, Nevada: Evidence for the coupling of hydrogeologic, diagenetic, and tectonic processes, Geological Society of America Bulletin 116 (September/October, 2004) 1120-1136.

R. Sternlof, Kurt, J. W. Rudnicki, D. D. Pollard, Anticrack inclusion model for compaction bands in sandstone., J. Geophys. Res. 110 (2005).

A. Aydin, R. Ahmadov, Bed-parallel compaction bands in aeolian sandstone: Their identification, characterization and implications, Tectonophysics 479 (2009) $277-284$. 
W. Taylor, D. Pollard, Estimation of in situ permeability of deformation bands in porous sandstone, Valley of Fire, Nevada, WATER RESOURCES RESEARCH 36 (2000) 2595-2606.

I. Main, K. Mair, O. Kwon, S. Elphick, B. Ngwenya, Experimental constraints on the mechanical and hydraulic properties of deformation bands in porous sandstones: a review, Geological Society, London, Special Publications 186 (2001) 43-63.

K. R. Sternlof, J. R. Chapin, D. D. Pollard, L. J. Durlofsky, Permeability effects of deformation band arrays in sandstone, AAPG Bulletin 88 (2004) 1315 - 1329.

R. Sternlof, Kurt, M. Karimi-Fard, D. D. Pollard, L. J. Durlofsky, Flow and transport effects of compaction bands in sandstone at scales relevant to aquifer and reservoir management, Water Resour. Res. 42 (2006).

W. Sun, J. E. Andrade, J. W. Rudnicki, P. Eichhubl, Connecting microstructural attributes and permeability from 3D tomographic images of in situ shear-enhanced compaction bands using multiscale computations, Geophys. Res. Lett. 38 (2011).

R. Katsman, E. Aharonov, A study of compaction bands originating from cracks, notches, and compacted defects, Journal of Structural Geology 28 (2005) 508 - 518.

R. Katsman, E. Aharonov, H. Scher, A numerical study on localized volume reduction in elastic media: Some insights on the mechanics of anticracks, J. Geophys. Res. 111 (2006a).

R. Katsman, E. Aharonov, H. Scher, Localized compaction in rocks: Eshelby's inclusion and the spring network model, J. Geophys. Res. Lett, 33-10, L10311. (2006b).

J. Rudnicki, J. Rice, Conditions for the localization of deformation in pressure-sensitive dilatant materials, Journal of the Mechanics and Physics of Solids 23 (1975) 371 - 394.

W. A. Olsson, Theoretical and experimental investigation of compaction bands in porous rock, J. Geophys. Res. 104 (1999) 7219 - 7228.

K. A. Issen, J. W. Rudnicki, Conditions for compaction bands in porous rock, J. Geophys. Res. 105 (2000).

T.-F. Wong, P. Baud, E. Klein, Localized failure modes in a compactant porous rock, J. Geophys. Res. 38 (2001).

P. Besuelle, Compacting and dilating shear bands in porous rock: Theoretical and experimental conditions, J. Geophys. Res. 106 (2001) 13435 - 13442.

R. I. Borja, A. Aydin, Computational modeling of deformation bands in granular media. i. geological and mathematical framework, Computer Methods in Applied Mechanics and Engineering 193 (2004) $2667-2698$.

J. W. Rudnicki, Shear and compaction band formation on an elliptic yield cap, J. Geophys. Res. 109 (2004).

K. A. Issen, V. Challa, Influence of the intermediate principal stress on the strain localization mode in porous sandstone, J. Geophys. Res. 113 (2008).

J. W. Rudnicki, K. R. Sternlof, Energy release model of compaction band propagation, J. Geophys. Res. 32 (2005).

J. W. Rudnicki, Models for compaction band propagation, chapter 8 in rock physics and geomechanics 
in the study of reservoirs and repositories, Geological Society, London, Special Publications (2007) $107-125$.

R. A. Schultz, Scaling and paleodepth of compaction bands, Nevada and Utah, J. Geophys. Res. 114 (2009).

S. Tembe, P. Baud, T.-F. Wong, Stress conditions for the propagation of discrete compaction bands in porous sandstone, J. Geophys. Res. 113 (2008).

P. Baud, W. Zhu, T.-F. Wong, Failure mode and weakening effect of water on sandstone, J. Geophys. Res. 105 (2000).

K. Mair, I. Main, S. Elphick, Sequential growth of deformation bands in the laboratory, Journal of Structural Geology 22 (2000) $25-42$.

W. A. Olsson, D. J. Holcomb, Compaction localization in porous rock, Geophys. Res. Lett. 17 (2000) $3537-3540$.

V. Vajdova, T.-F. Wong, Incremental propagation of discrete compaction bands: Acoustic emission and microstructural observations on circumferentially notched samples of Bentheim, Geophys. Res. Lett. 30 (2003).

H. Tada, P. Paris, G. Irwin, Stress Analysis of Cracks Handbook, Third Edition, ASME International, 2000.

S. Deng, A. Aydin, Distribution of compaction bands in 3D in an aeolian sandstone: The role of cross-bed orientation, Tectonophysics 574575 (2012) $204-218$.

J. D. Eshelby, The determination of the elastic field of an ellipsoidal inclusion, and related problems, Proceedings of the Royal Society of London. Series A. Mathematical and Physical Sciences 241 (1957) 376-396.

J. D. Eshelby, The elastic field outside an ellipsoidal inclusion, Proceedings of the Royal Society of London. Series A. Mathematical and Physical Sciences 252 (1959) 561-569.

J. D. Eshelby, Elastic inclusions and inhomogeneities, Progress in solid mechanics 2 (1961) 89-140.

Z. Reches, Tensile fracturing of stiff rock layers under triaxial compressive stress states, Int. J. of Rock Mech. and Min. Sci 35:4-5 (1998).

A. Eidelman, Z. Reches, Fractured pebbles-a new stress indicator, Geology 20 (1992) 301-304.

J. W. Rudnicki, The inception of faulting in a rock mass with a weakened zone, J. Geophys. Res. 82:5 (1977).

J. W. Rudnicki, Alteration of regional stress by reservoirs and other inhomogeneities: Stabilizing or destabilizing?, Proceedings of the Ninth International Congress on Rock Mechanics, Paris 3 (1999) $1629-1637$.

D. Healy, R. Jones, R. Holdsworth, Three-dimensional brittle shear fracturing by tensile crack interaction, Nature 439 (2006a) 64-67.

D. Healy, R. Jones, R. Holdsworth, New insights into the development of brittle shear fractures from a 3-d numerical model of microcrack interaction, Earth and Planetary Science Letters 249 (2006b) 14-28. Cited By (since 1996)13.

C. Meng, F. Maerten, D. Pollard, Modeling mixed-mode fracture propagation in isotropic elastic three 
dimensional solid, International Journal of Fracture 179 (2013) 45-57.

D. Healy, Short note: Elastic field in 3D due to a spheroidal inclusion-MATLAB code for Eshelby's solution, Comput. Geosci. 35 (2009) 2170-2173.

C. Meng, W. Heltsley, D. D. Pollard, Evaluation of the Eshelby solution for the ellipsoidal inclusion and heterogeneity, Computers and Geosciences (2011).

M. Cocco, J. R. Rice, Pore pressure and poroelasticity effects in coulomb stress analysis of earthquake interactions, Journal of Geophysical Research: Solid Earth 107 (2002) ESE 2-1-ESE 2-17.

T. Mura, Micromechanics of defects in solids, Mechanics of elastic and inelastic solids, Kluwer Academic Pub., 1987.

M. Igor, Elliptic integrals and functions, MathWork (2005).

B. C. Haimson, Borehole breakouts in berea sandstone reveal a new fracture mechanism, Pure and Applied Geophysics 160 (2003) 813-831. 10.1007/PL00012567.

D. D. Pollard, R. C. Fletcher, Fundamentals of Structural Geology, Cambridge University Press, 2006.

P. Avseth, O. Petroleum, T. Mukerji, G. Mavko, Quantitative Seismic Interpretation:Applying Rock Physics Tools to Reduce Interpretation Risk., Cambridge University Press, 2005.

R. Burgmann, D. D. Pollard, S. J. Martel, Slip distributions on faults: effects of stress gradients, inelastic deformation, heterogeneous host-rock stiffness, and fault interaction, Journal of Structural Geology 16 (1994) $1675-1690$.

F. Gassmann, ber die elastizitt porser medien, Viertel. Naturforsch. Ges. Zrich 96 (1951) 1-23.

Z. Bieniawski, Rock mechanics design in mining and tunneling, A.A. Balkema, 1984.

M. Williams, On the stress distribution at the base of a stationary crack, J. Appl. Mech.,24 (1957), p. 109 (1957).

B. R. Lawn, T. R. Wilshaw, Fracture of brittle solids, Cambridge University Press, 1975.

V. F. Erdogan, G. C. Sih, On the crack extension in plates under plane loading and transverse shear, Trans ASME J Bas Eng 85D (1963) 519.

G. U. Yule, An introduction to the theory of statistics, London, C. Griffin and company, limited, 1919. 


\section{A}

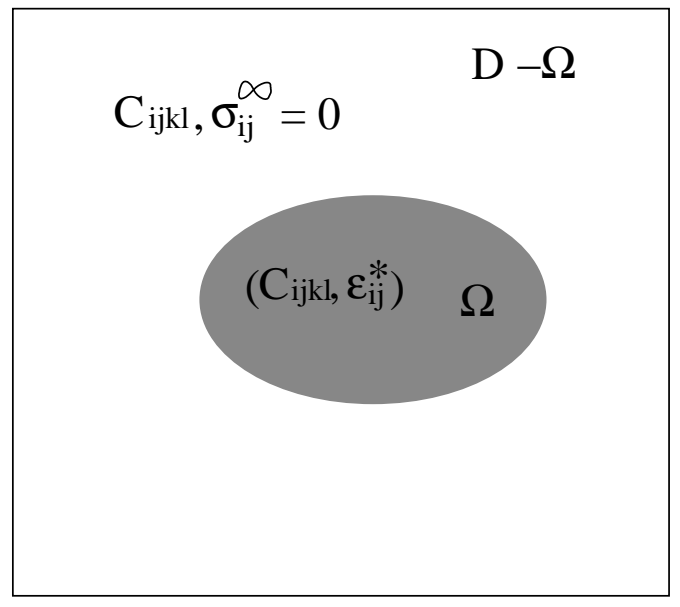

\section{B}

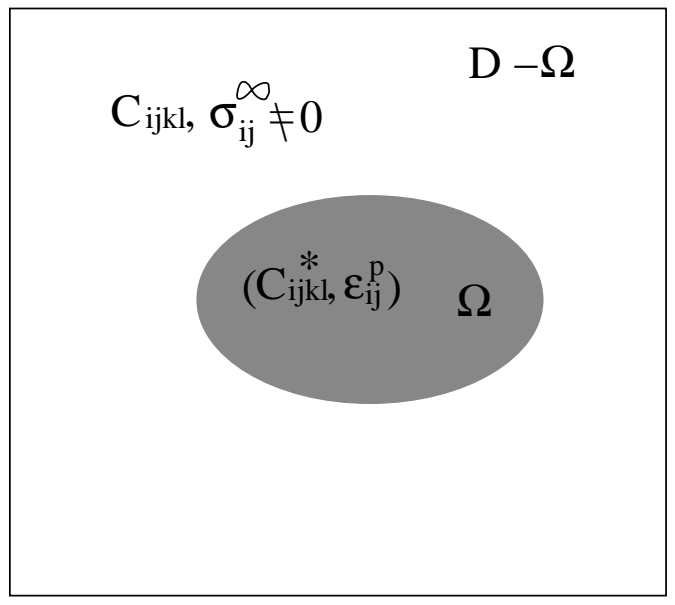

Figure 1: A, inclusion problem: uniform elastic moduli, zero remote stress, inclusion $\Omega$ under eigenstrain $\epsilon^{*} ; \mathbf{B}$, inhomogeneous inclusion problem: different elastic moduli, non-zero remote stress, initial eigenstrain $\epsilon^{p}$ in $\Omega$. 


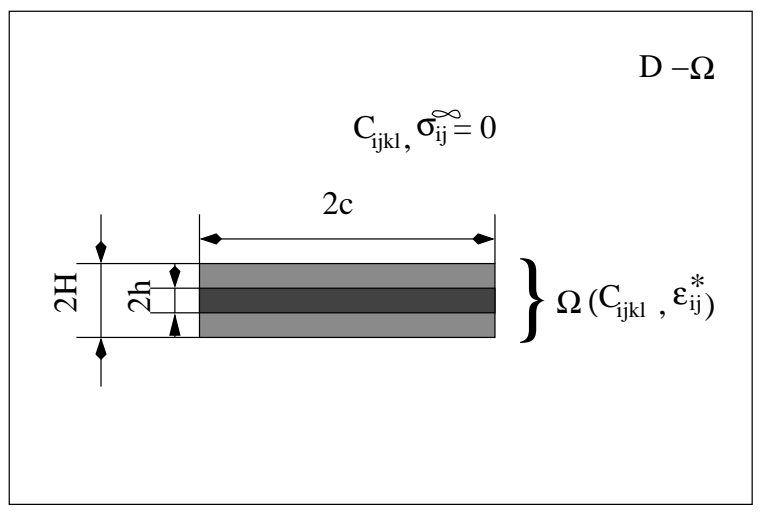

Figure 2: Compaction band modeled as a $2 \mathrm{D}$ rectangular inclusion with thickness reduced by $2(H-h)$.

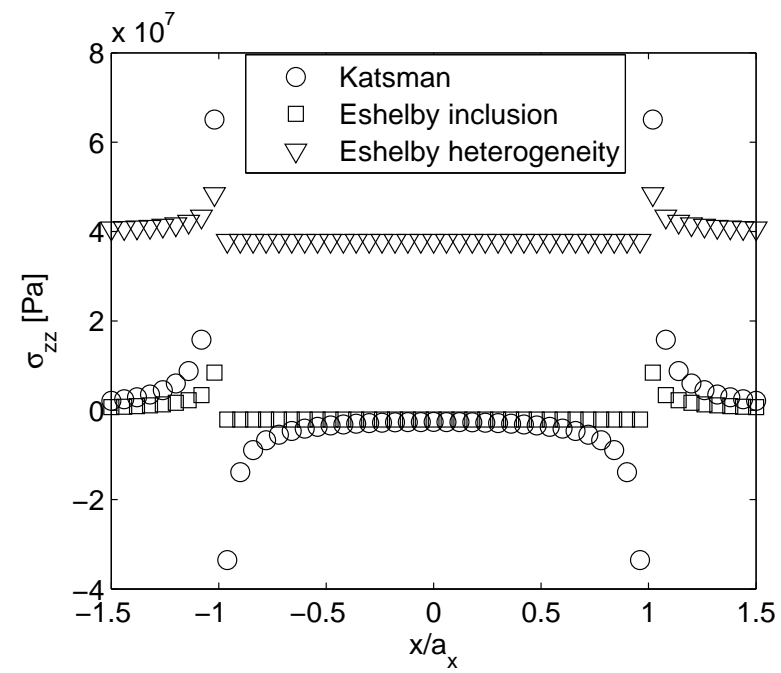

Figure 3: Normal stress $\sigma_{z z}$ along the $x$ axis by Katsman et al. (2006b), Eshelby's solution for inclusion, and Eshelby's solution for inhomogeneous inclusion. 

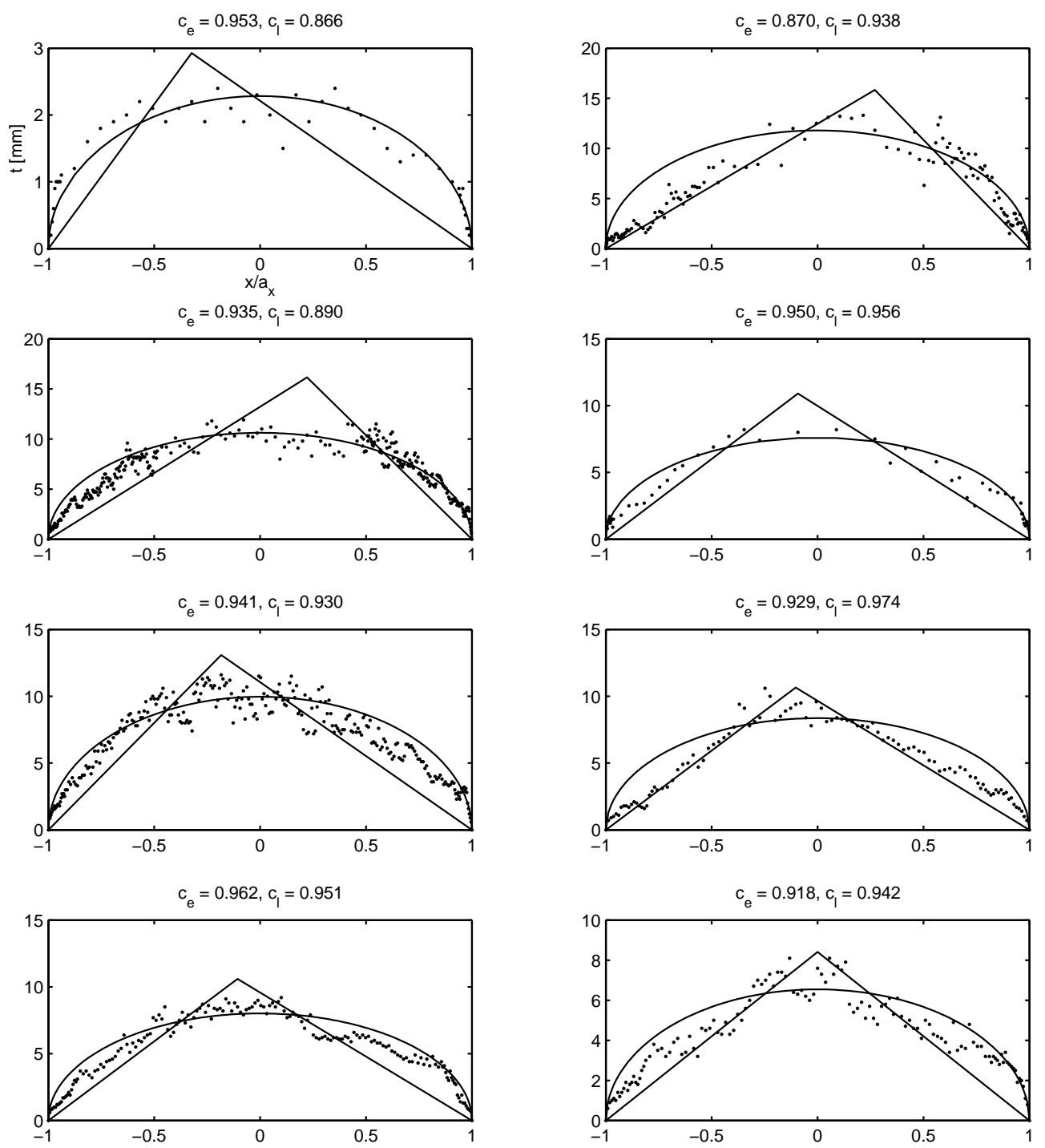

Figure 4: Compaction band thickness $t$ as a function of normalized length $x / a_{x}$; elliptical and piece-wise linear fits for the thickness-length relation and associated correlation coefficients, $c_{e}$ and $c_{l}$ (Yule (1919)). 

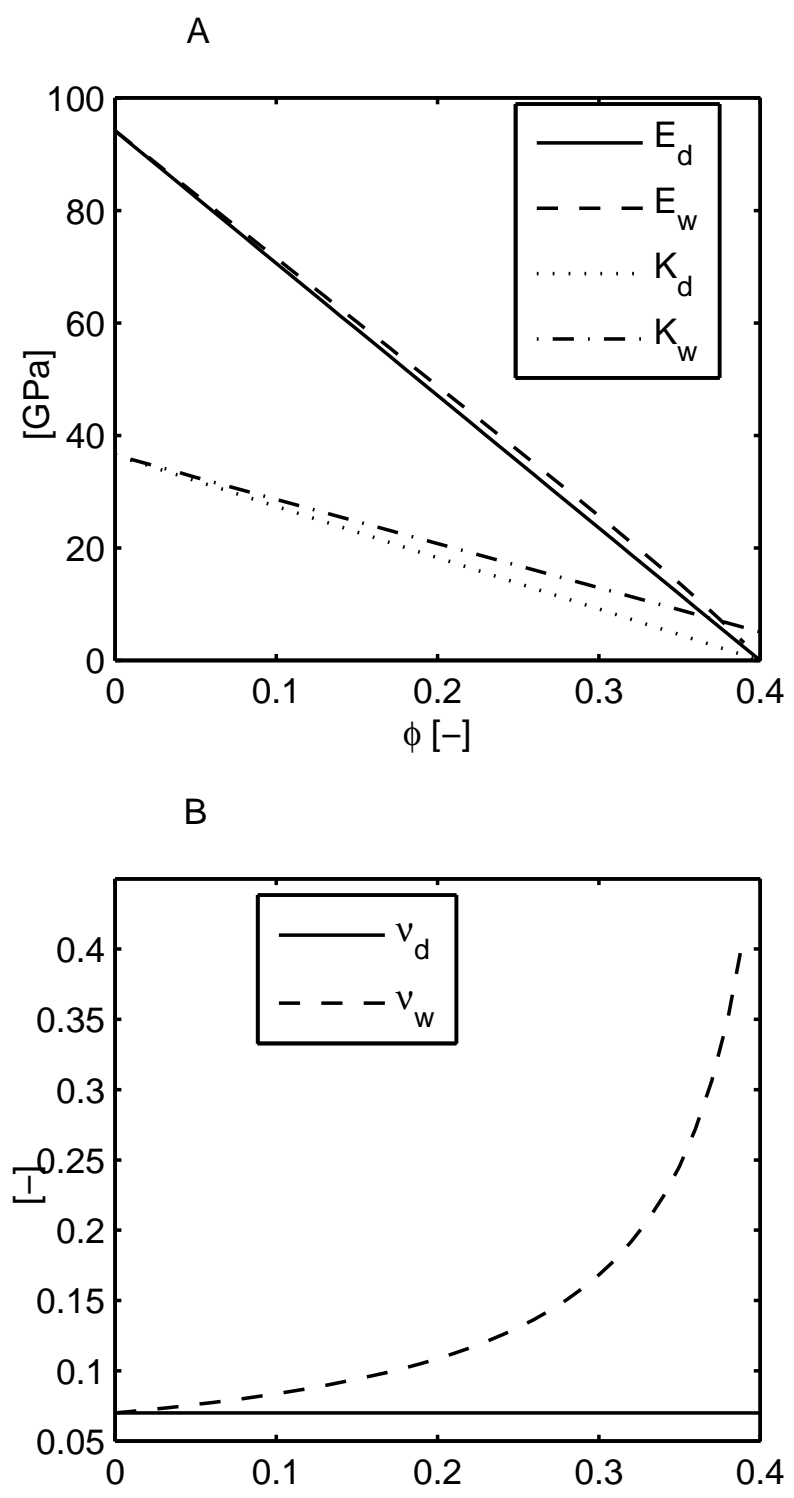

Figure 5: A, Bulk modulus $K_{d(w)}$ and Young's modulus $E_{d(w)}$ plotted versus porosity $\phi$; B, Poisson's ratio $\nu_{d(w)}$ versus $\phi$ for dry (d) and wet (w) sandstones. 


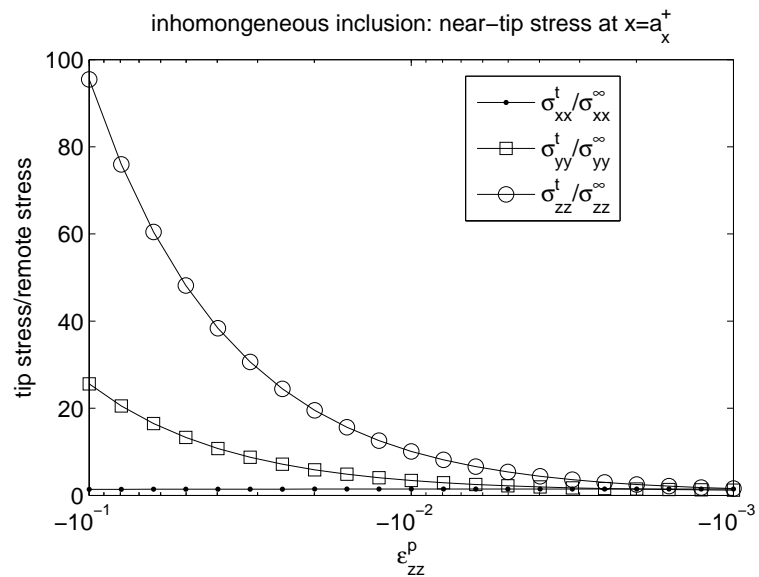

Figure 6: Near-tip stress components plotted versus the plastic strain for the penny-shaped inhomogeneity with model parameters defined in the text.

A

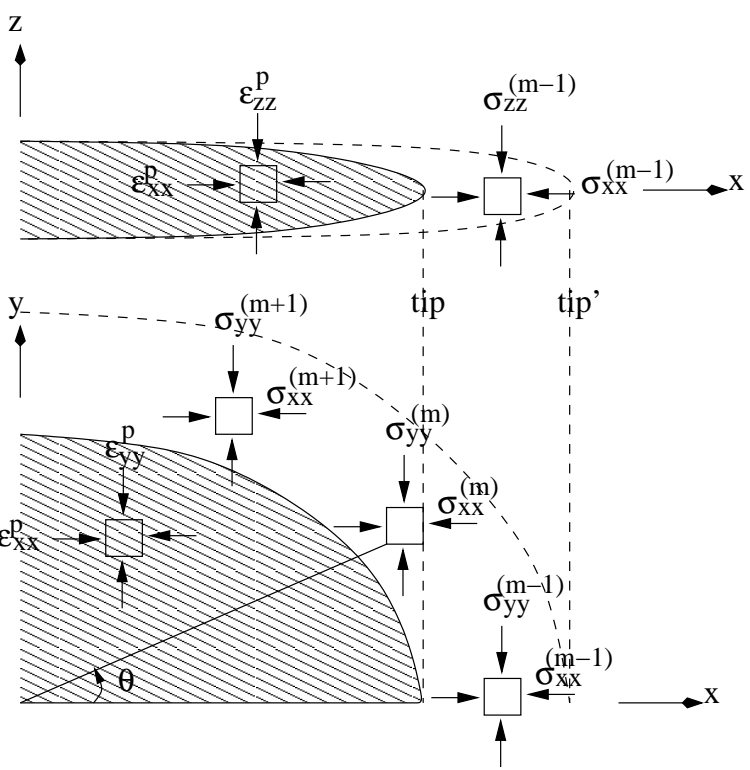

Figure 7: Cross-section of the model compaction band: $\mathbf{A},(x, z)$-plane; $\mathbf{B},(x, z)$-plane. When the band tip-line advances, marked by the dashed lines, the tip-incipient areas under stress $\sigma_{i j}^{m}$ will join the compaction band by undergoing a plastic strain $\epsilon_{i j}^{p}$. 


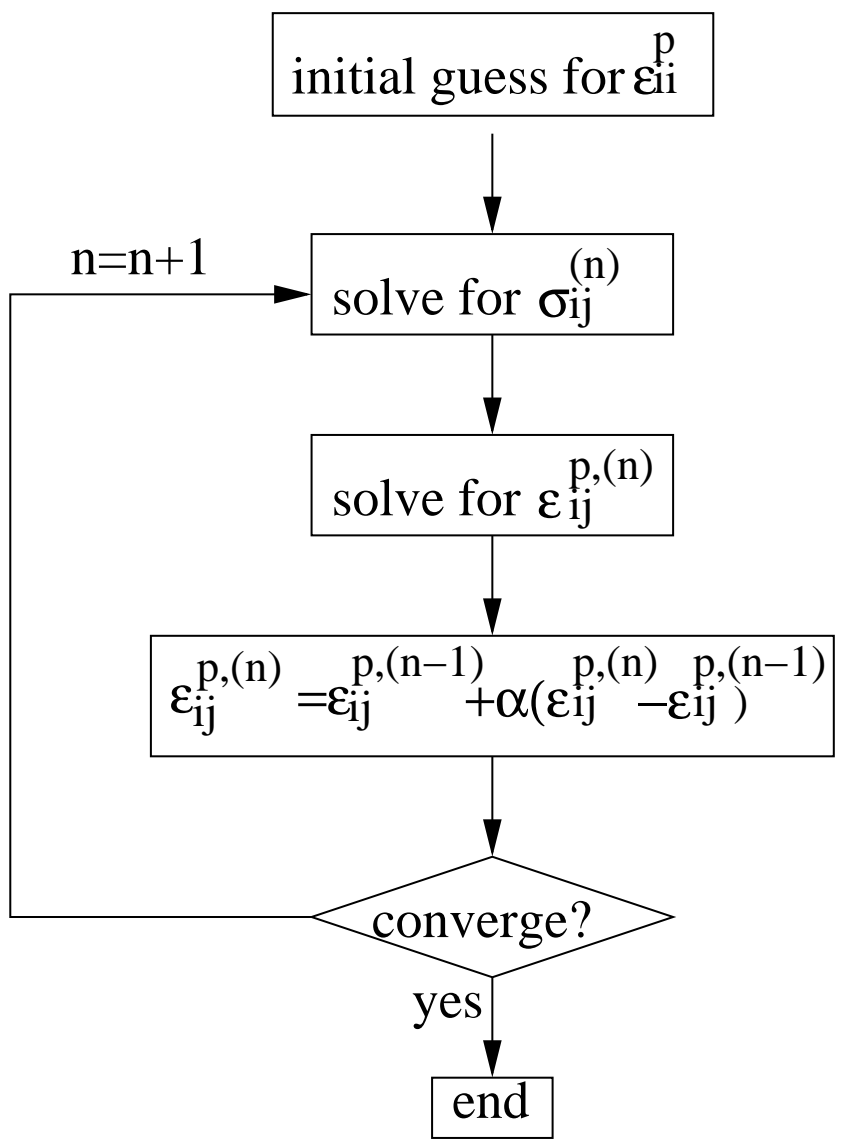

Figure 8: Iterative scheme resolving the tip-incipient stress $\sigma_{i j}$ and plastic strain $\epsilon_{i j}^{p}$. 


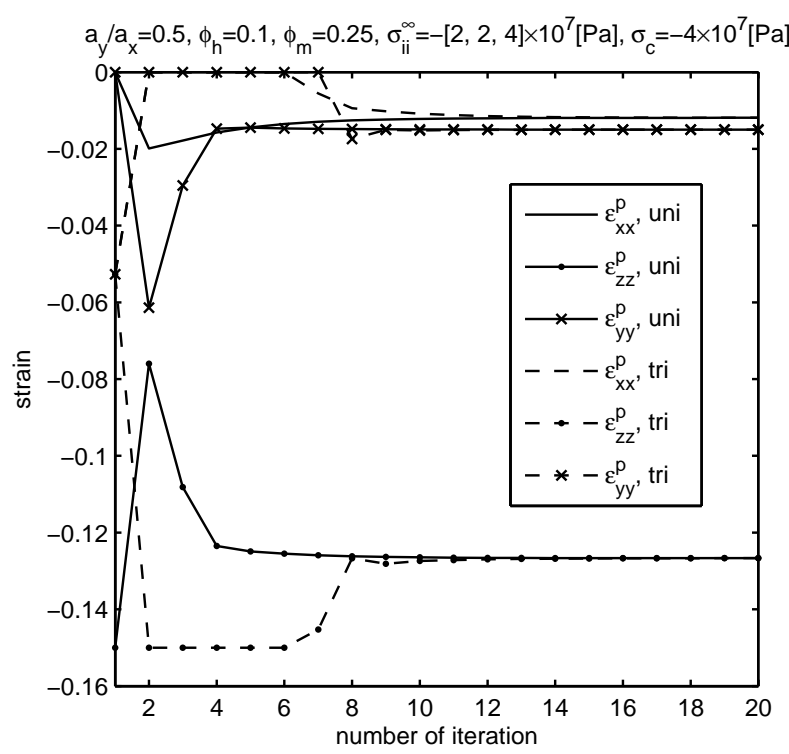

Figure 9: Convergent behaviors of the three principal plastic strains $\epsilon_{i i}^{p}$ for different guesses, i.e. uniaxial and triaxial. 

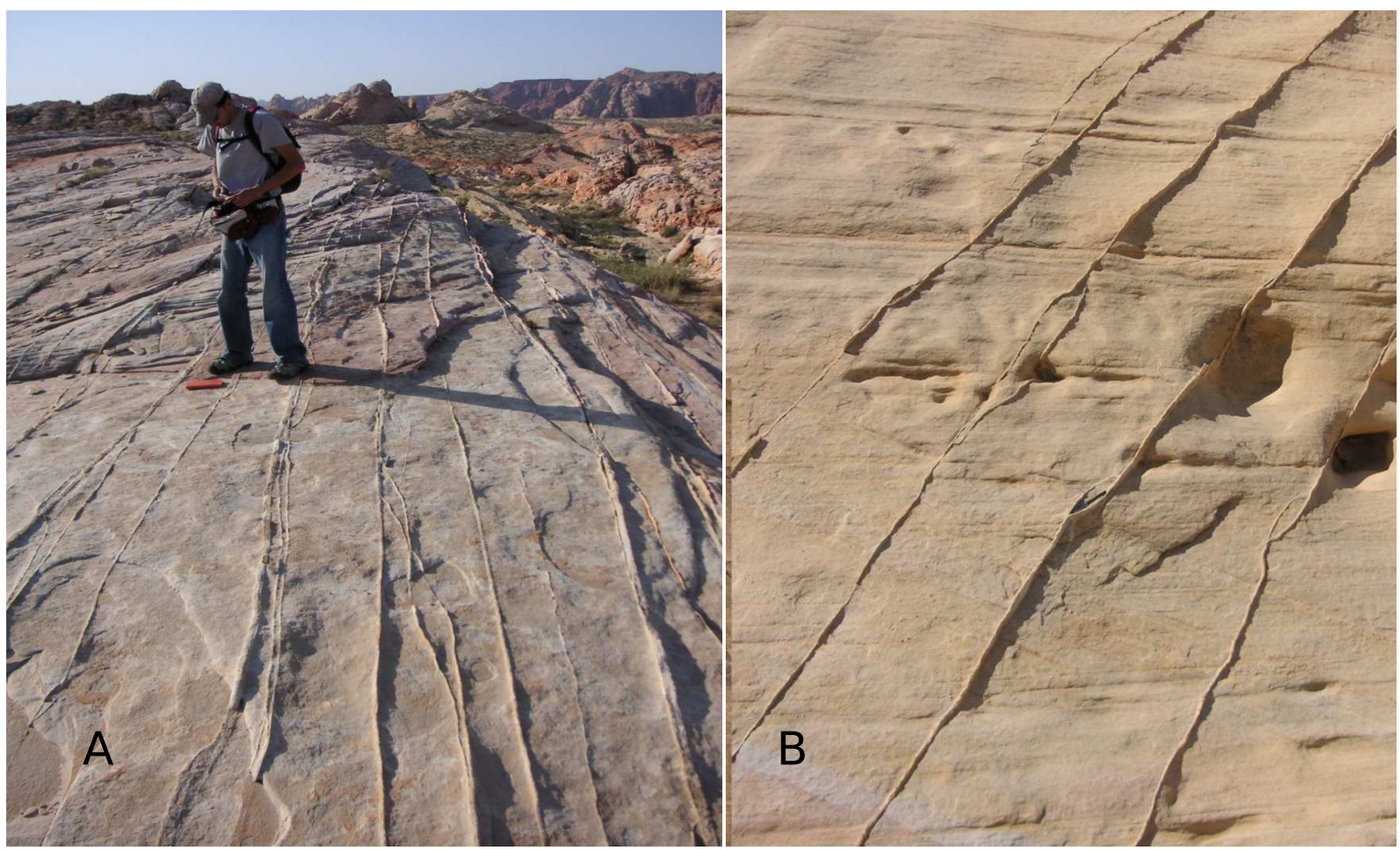

Figure 10: Pure compaction bands at Valley of Fire State Park, NV (Sternlof et al. (2005)): A, subparallel set with some anastamosing: typical spacing is of several decimeter, but some are very closely spaced; B, sub-parallel compaction bands with eye-structures. 

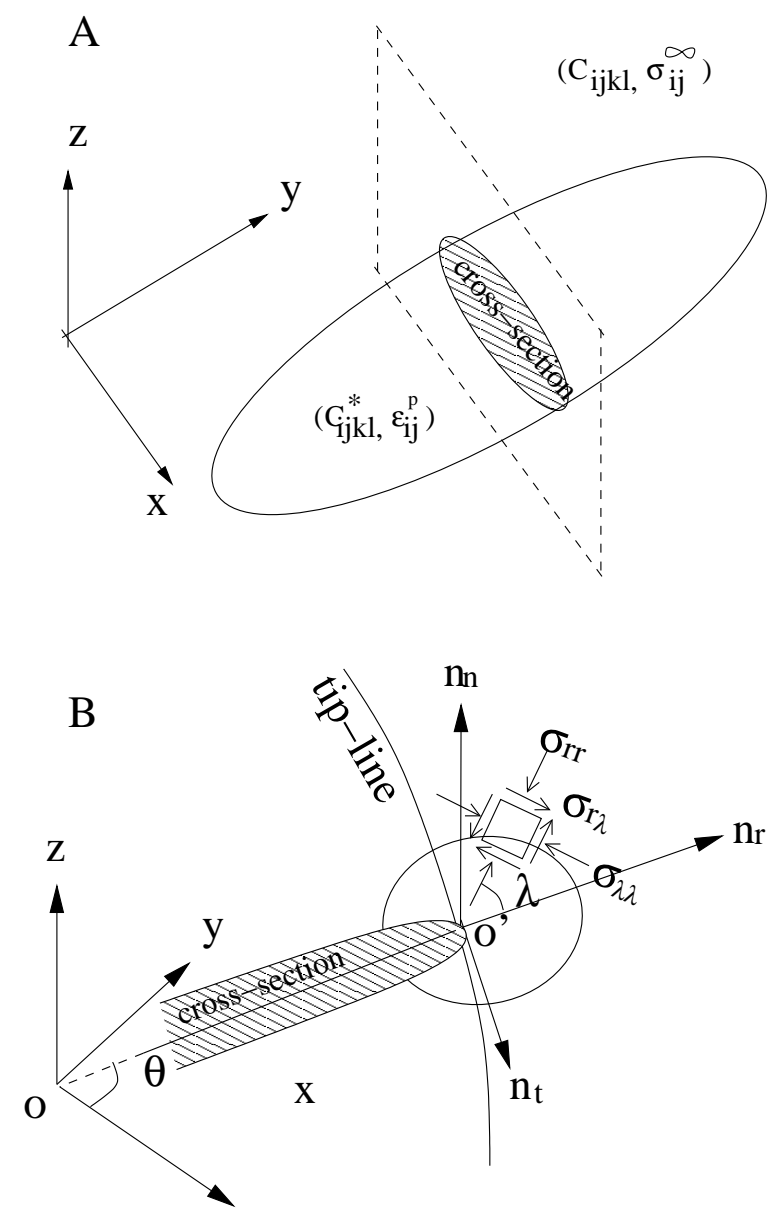

Figure 11: A, Cartesian coordinates on cross-section planes of the ellipsoidal inhomogeneous inclusion. $\mathbf{B}$, polar coordinate $(\lambda, r)$ about the ellipsoid tip-line at longitude angle $\theta$. 

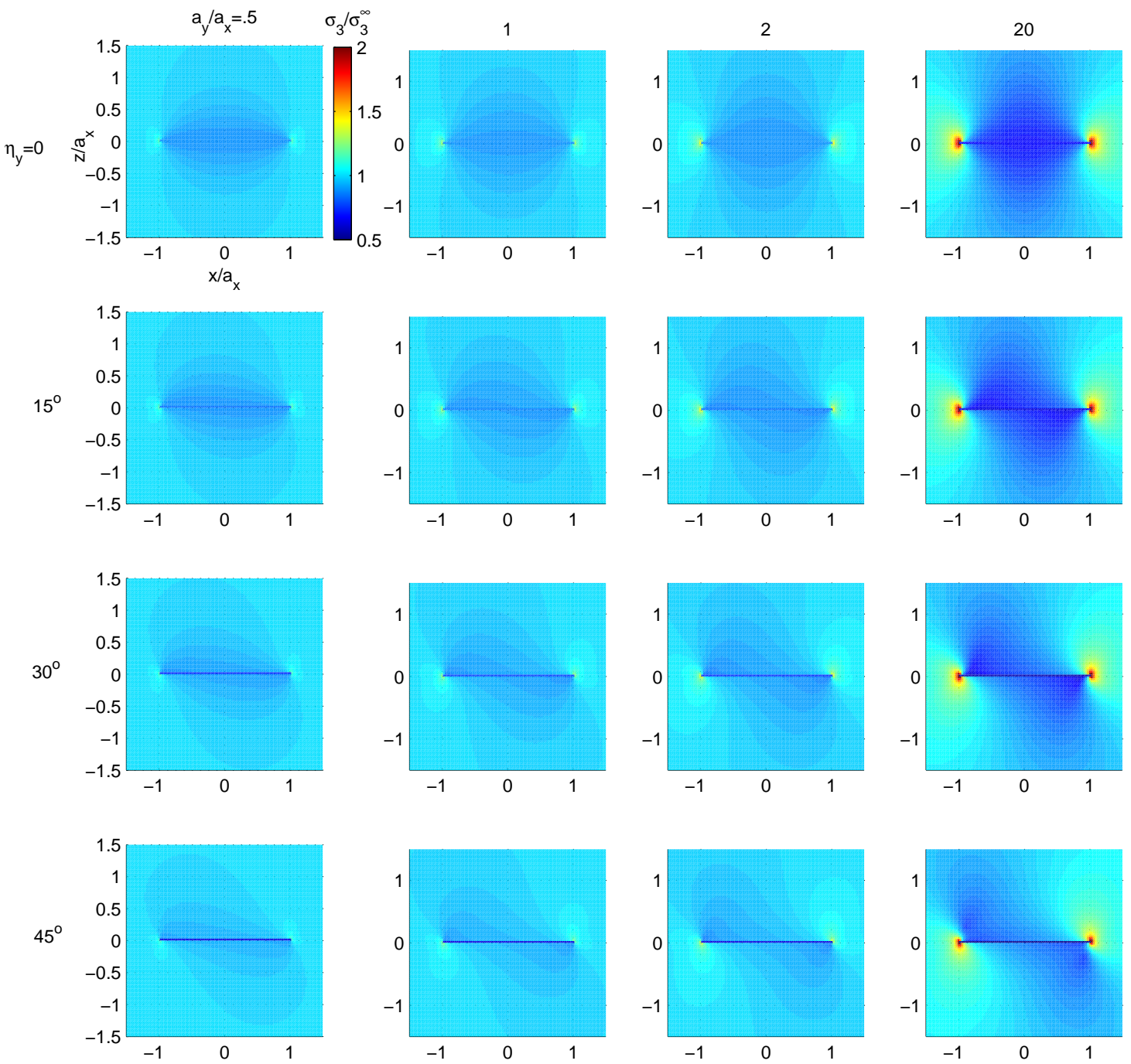

Figure 12: Normalized principal stress $\sigma_{3} / \sigma_{3}^{\infty}$ evaluated the cross-section plane $y=0$ for axial-ratio $a_{y} / a_{x}=\left[\begin{array}{llll}0.5 & 1 & 2 & 20\end{array}\right]$ (column), under a pure compressive remote stress $\left(\eta_{y}=0\right)$, and under that stress rotated around $y$-axis by $\eta_{y}=\left[\begin{array}{lll}15 & 30 & 45\end{array}\right]^{\circ}$ (row). 

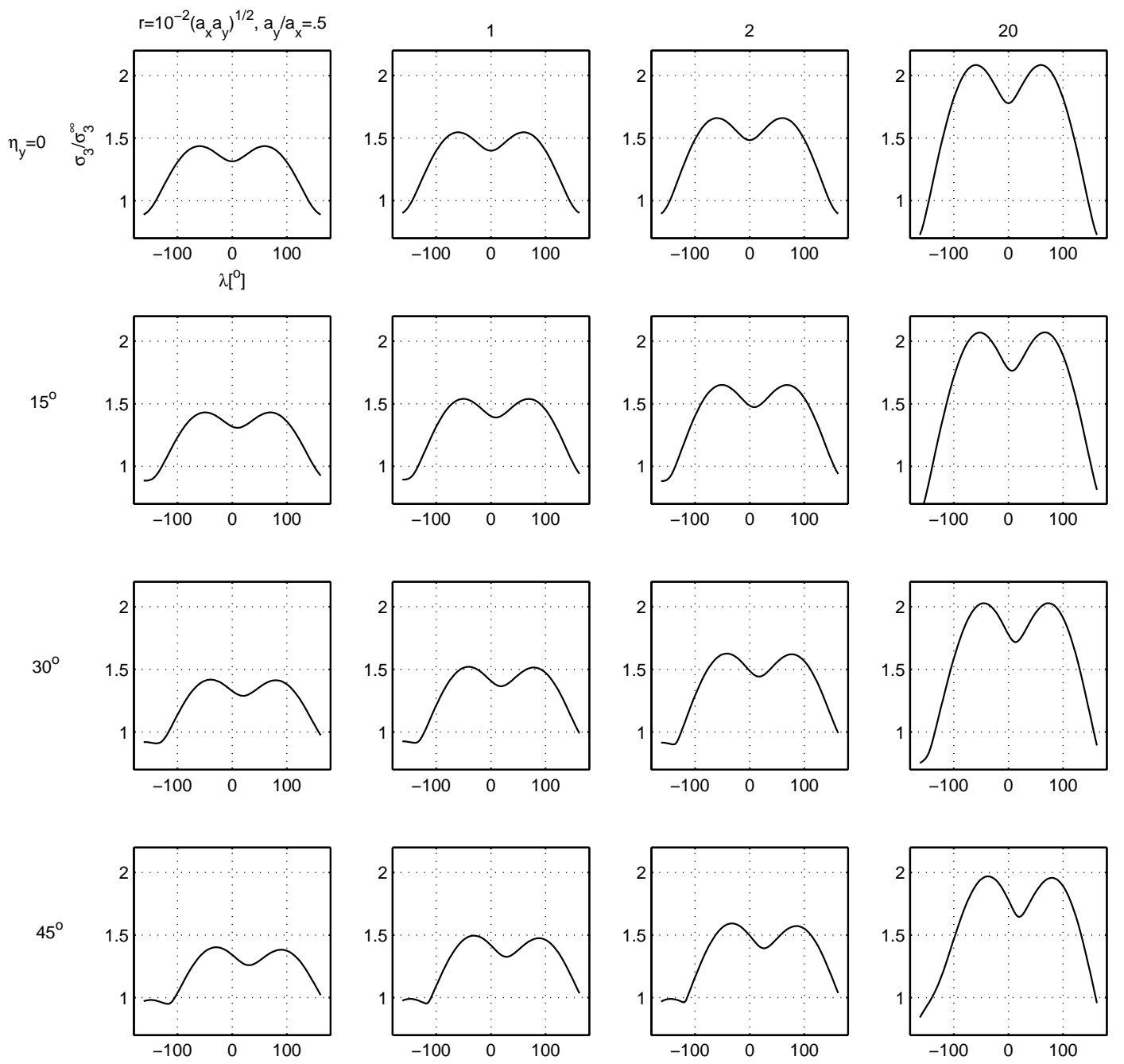

Figure 13: Normalized principal stress $\sigma_{3} / \sigma_{3}^{\infty}$ evaluated on a circle $\left(r=10^{-2} \sqrt{a_{x} a_{y}}, \lambda\right)$ around the tip

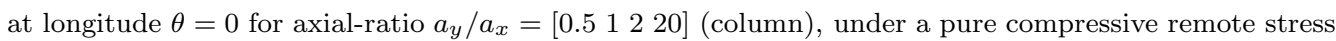
$\left(\eta_{y}=0\right)$, and under that stress rotated around $y$-axis by $\eta_{y}=\left[\begin{array}{lll}15 & 30 & 45\end{array}\right]^{\circ}$ (row). 


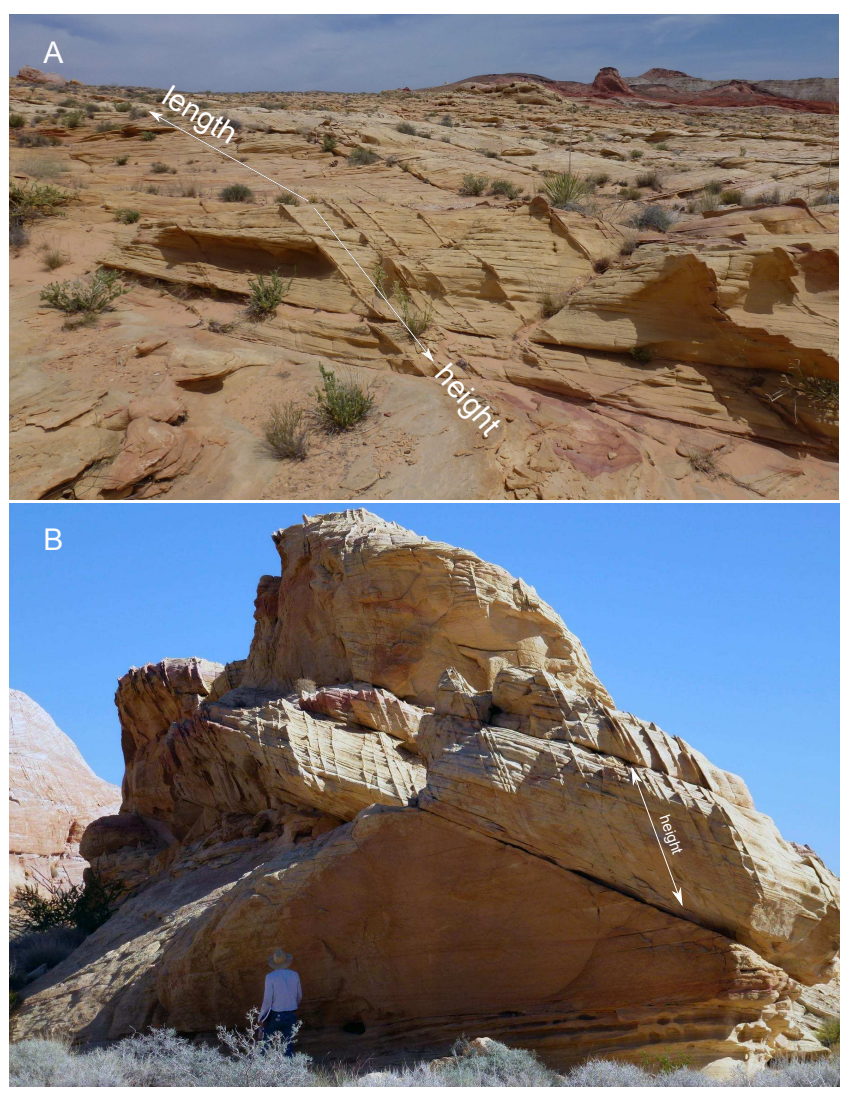

Figure 14: Two examples of compaction bands constrained in height by dune boundaries at the Valley of Fire, NV. When the band heights are limited by the dune boundary, the regions of influence are mainly determined the heights rather than the lengths. 


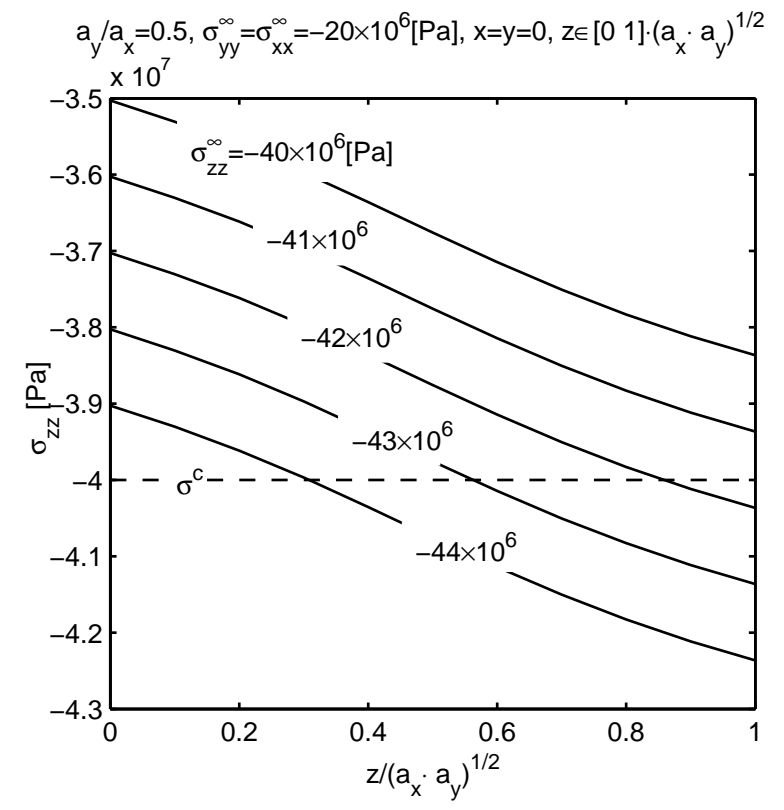

Figure 15: Normal stress $\sigma_{z z}$ evaluated along $z$-axis when the remote normal stress $\sigma_{z z}^{\infty}$ increases by up to $10 \%$ 

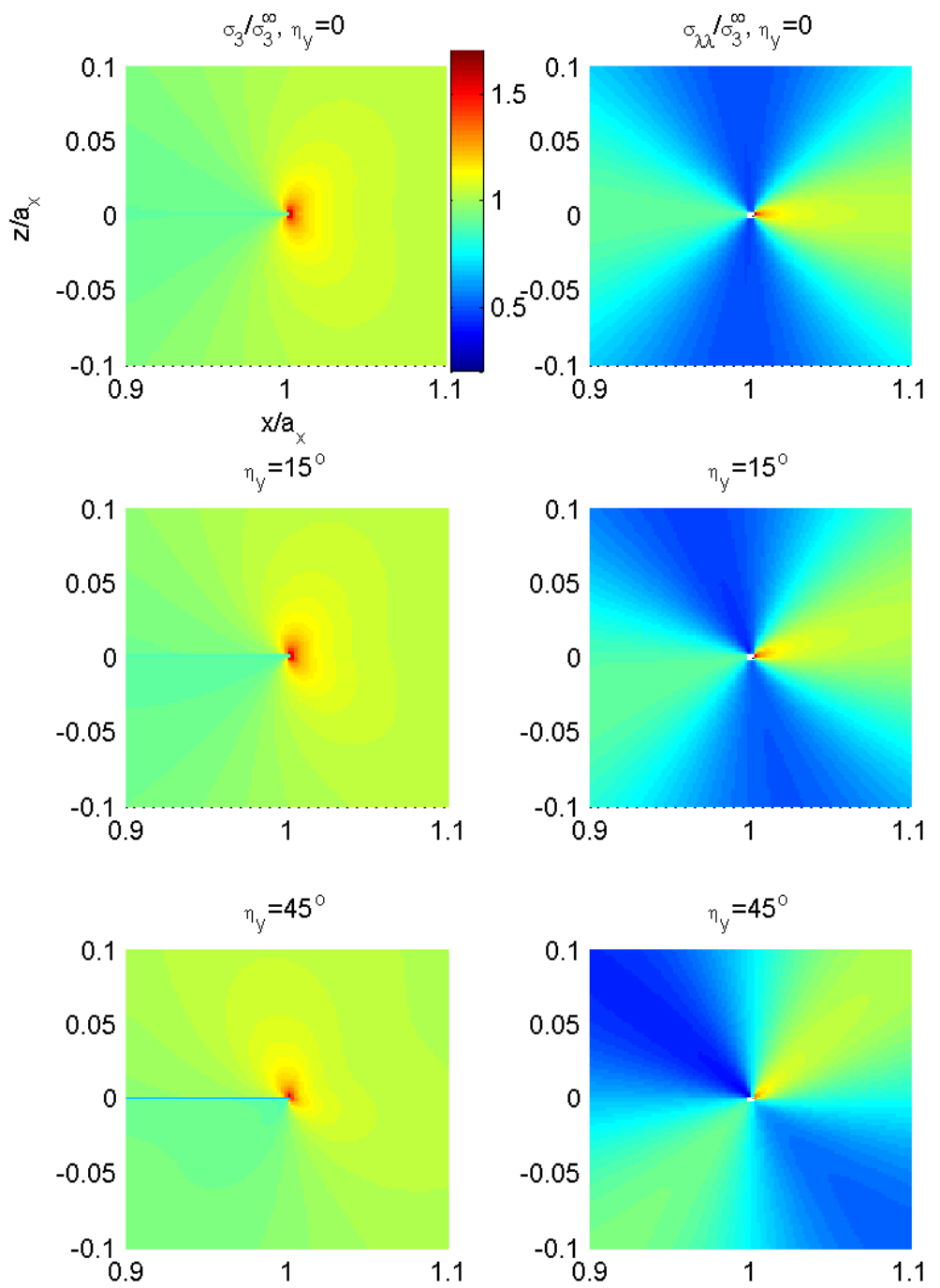

Figure 16: Normalized major principal stress $\sigma_{3} / \sigma_{3}^{\infty}$ and circumferential stress $\sigma_{\lambda \lambda} / \sigma_{3}^{\infty}$ evaluated on the near-tip grid about $\left[\begin{array}{lll}a_{x} & 0 & 0\end{array}\right]$ (i.e. an end of the long semi axis) under remote stress $\sigma_{i i}^{\infty}=-\left[\begin{array}{lll}20 & 20 & 40\end{array}\right]$ $\mathrm{MPa}$ and under this stress rotated around the $y$-axis by angle $\eta_{y}=\left[15^{\circ} 45^{\circ}\right]$. 

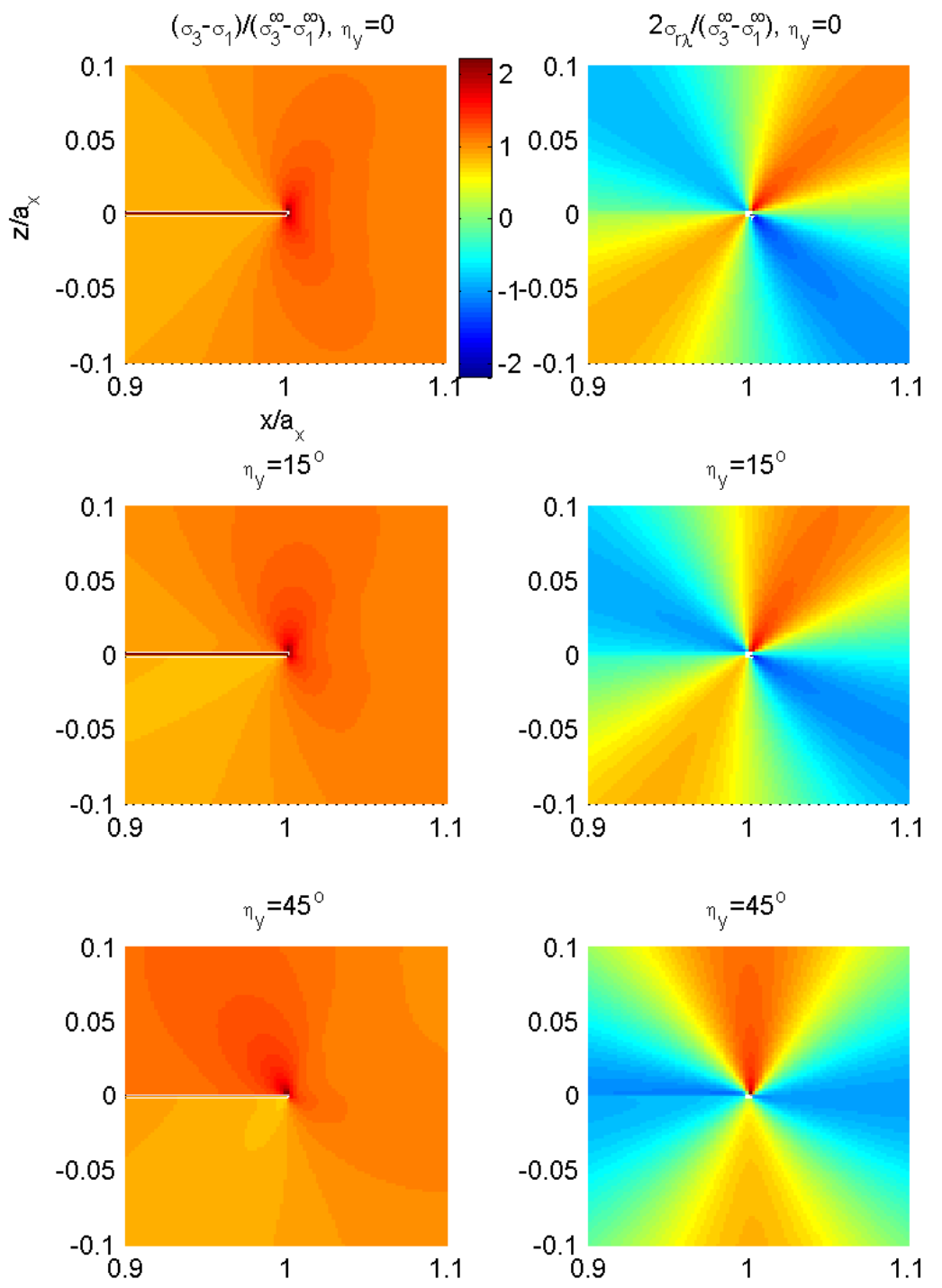

Figure 17: Near-tip maximum shear stresses (left) and circumferential shear stresses (right) normalized by remote maximum shear stress under remote stress $\sigma_{i i}^{p}=-\left[\begin{array}{lll}20 & 20 & 40\end{array}\right] \mathrm{MPa}$ and under this stress rotated about $y$-axis for angle $\eta_{y}=\left[\begin{array}{ll}15^{\circ} 45^{\circ}\end{array}\right]$. 

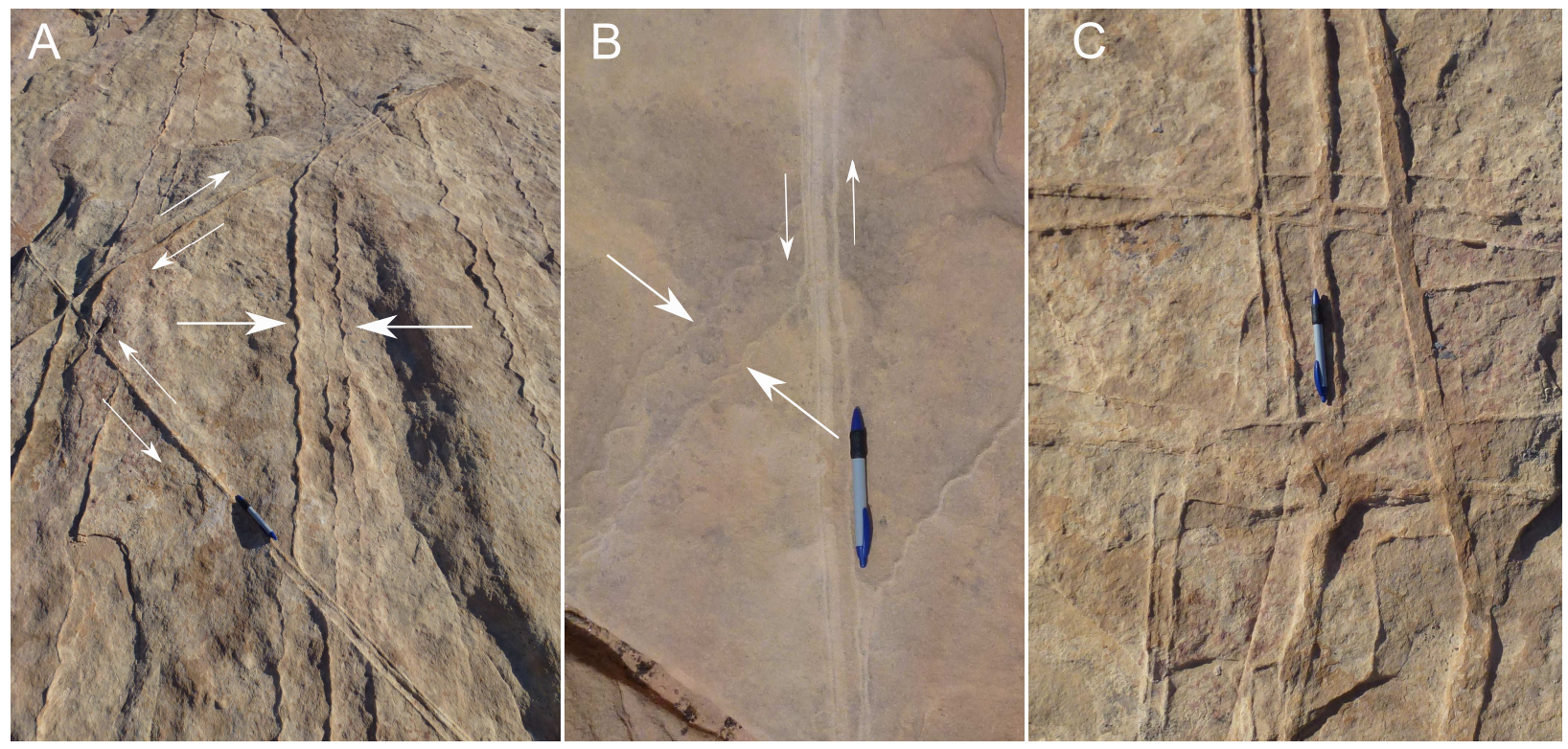

Figure 18: Field examples of compaction bands at the Valley of Fire, NV, that suggest all sets were active at the same time and under the same remote stress state. A, Wavy compaction bands (large arrows indicating the compaction directions) and shear-enhanced compaction bands (small arrow indicating the shear directions); B, Mutual joining relations for one set of shear-enhanced bands and wavy bands indicate both were active under the same stress field; C, Mutual joining relations for two sets of shearenhanced bands suggest both were active under the same stress field. 

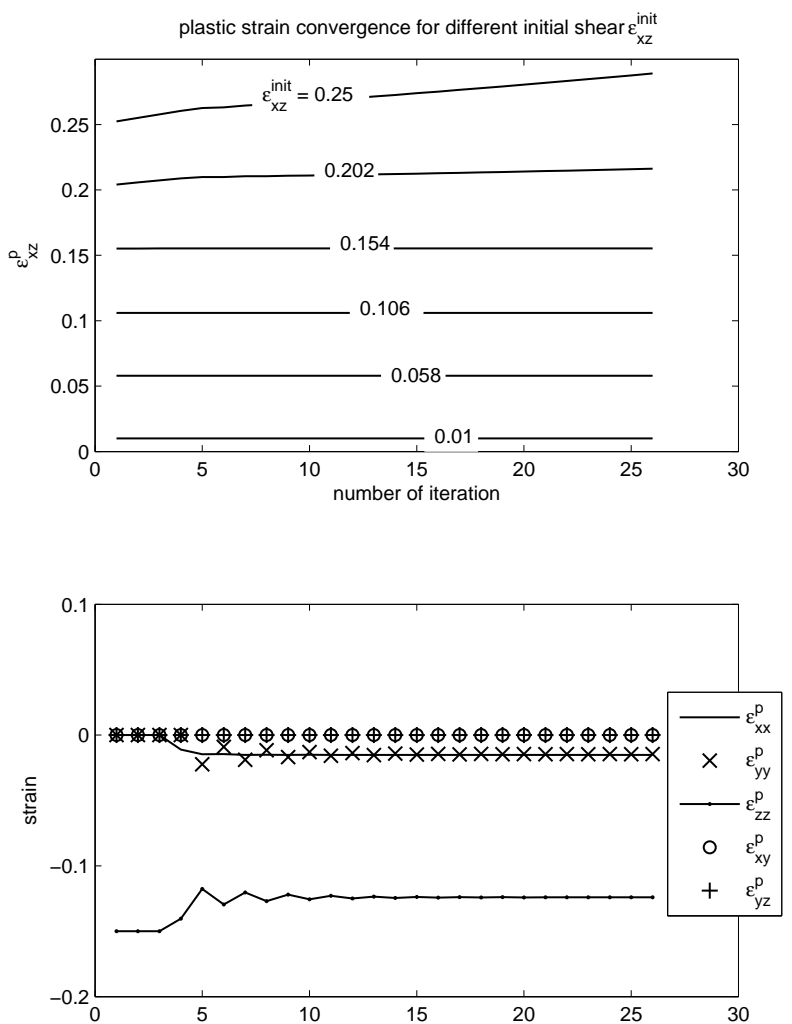

Figure 19: Upper: for initial in-band shear strain $\epsilon_{x z}^{p(0)} \in[0.01,0.25]$, evolution of the shear strain $\epsilon_{x z}^{p}$ as a function of the iteration number $n$. Lower: convergence of other components of $\epsilon_{i j}^{p}$ that are independent to the initial shear strain. 


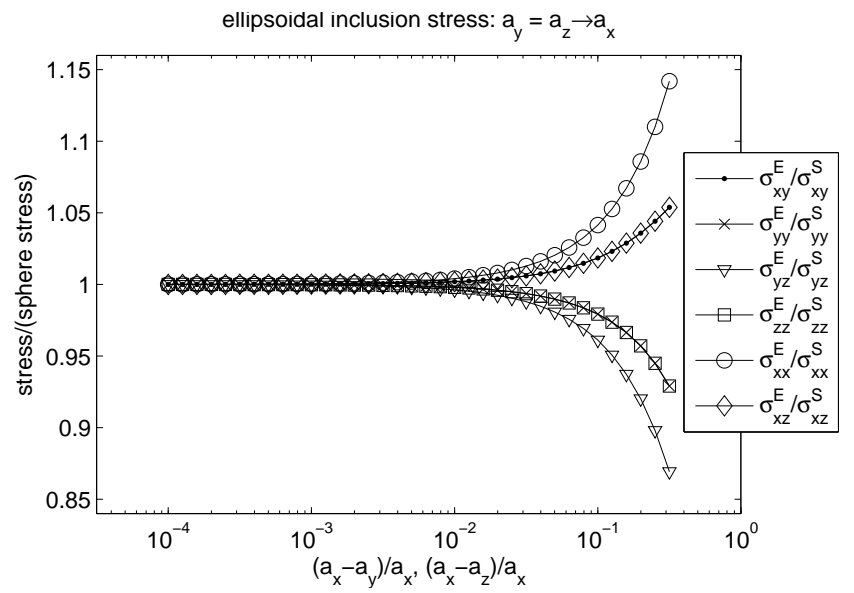

Figure 20: Normalized stress components inside ellipsoidal inclusion plotted versus normalized semi-axial differences.

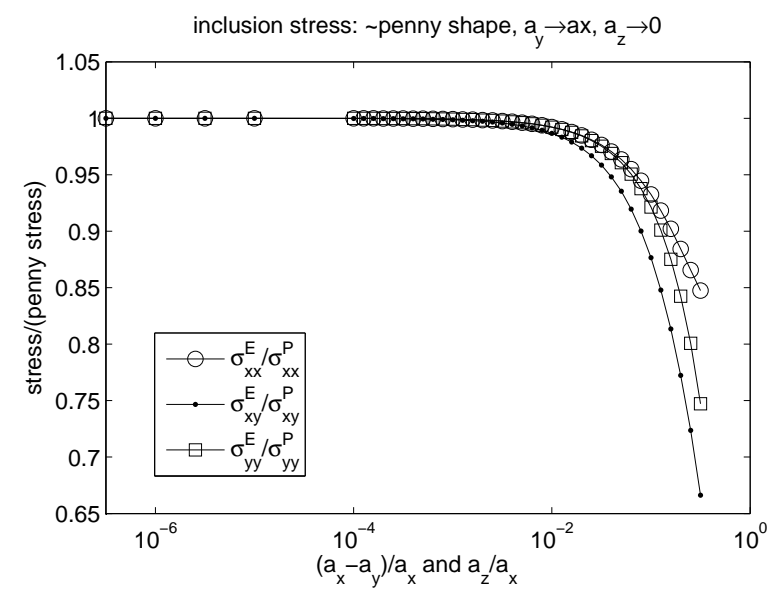

Figure 21: Normalized stress components inside ellipsoidal inclusion plotted versus the normalized semiaxial difference and normalized minor semi-axis as ellipsoid approaches the penny-shape. 


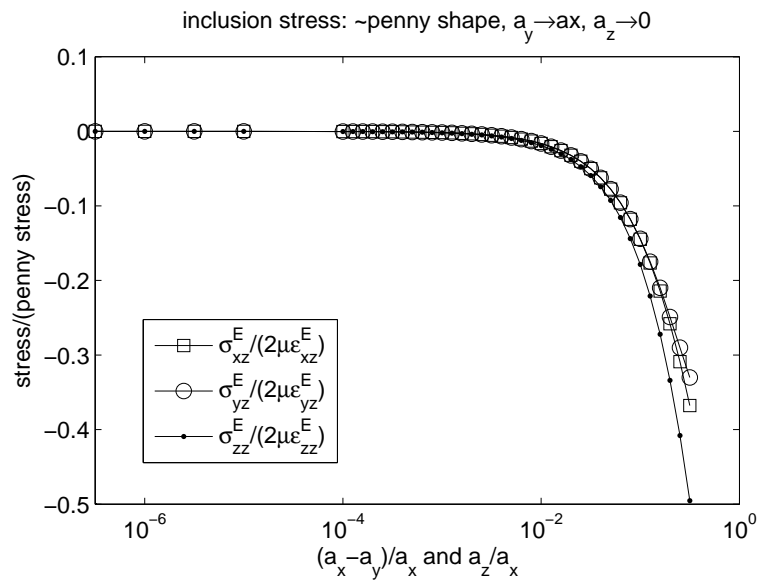

Figure 22: Normalized stress components inside ellipsoidal inclusion plotted versus normalized semi-axial difference and normalized minor axis.

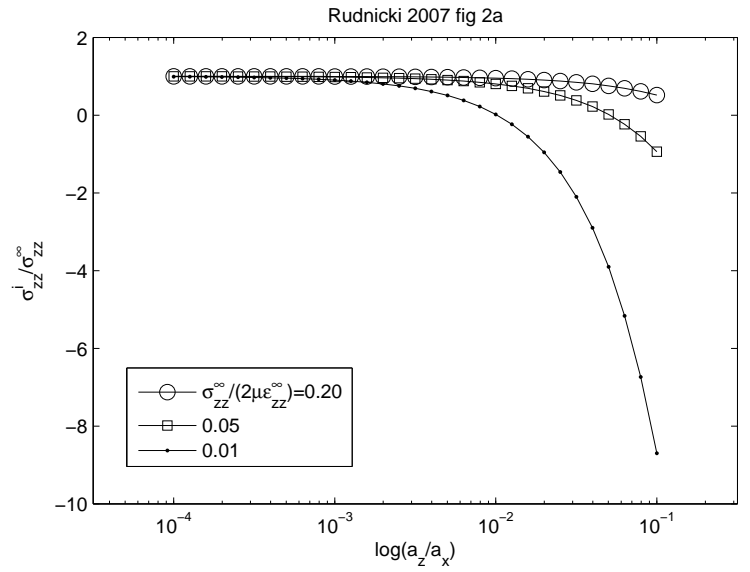

Figure 23: Normalized stress inside the axisymmetric ellipsoid versus aspect ratio (Rudnicki (2007),

Fig. 2a). The eigenstrain varies at constant remote stress and elastic shear modulus. 


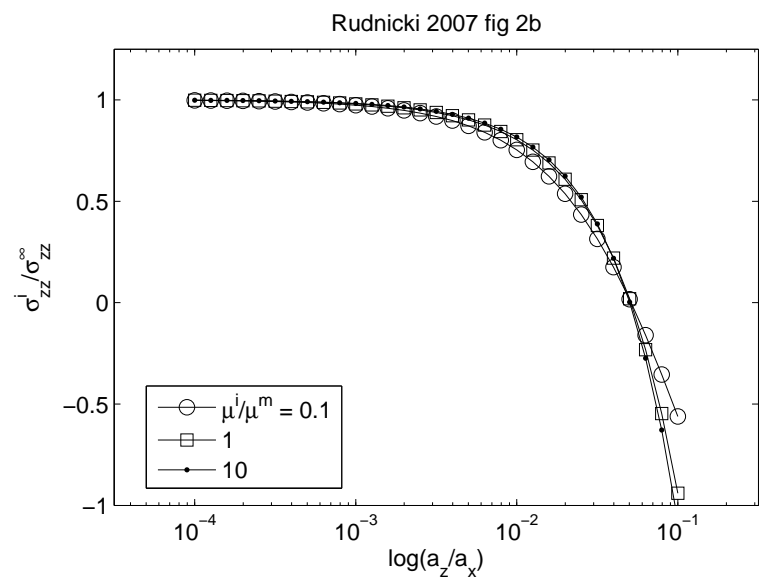

Figure 24: Normalized stress inside the axisymmetric ellipsoid versus aspect ratio (Rudnicki (2007), Fig. 2b). The ratio of shear modulus in the ellipsoid to that in the matrix is varied. 